Apidologie, 1973, 4 (4), 341-370.

\title{
LA THERMORÉGULATION ET L'ÉCOLOGIE DE QUELQUES ESPËCES D'ABEILLES SOCIALES D'AFRIQUE (APIDAE, TRIGONINI ET APIS MELLIFICA VAR. ADANSONII) ${ }^{\star}$
}

\author{
Wärmeregulierung und Ökologie einiger sozialer Bienen Afrikas \\ (Apidae, Trigonini und Apis mellifica var. adansonii)
}

\section{R. DARCHEN}

Station biologique, Faculté des Sciences de Paris 246:0 Les Eyzies

\section{SUMMARY}

THE THERMOREgULATION AND THE ECOLOGY OF SOME SOCIAL AFRICAN BEES (Apidae, Trigonini and Apis mellifica var. adansonii).

We have attempted to discover if there exist correlations between the ability to control the temperature of the hive and the ecology of nesting in some stingless bees and Apis mellifica var. adansonii.

First we describe the different biotopes of the african countries where this study was performed. Some stingless bees have successfully colonised the two depicted biotopes, the forest of Gabon (Makokou) and the savannah of the Ivory Coast (Lamto) thanks to their aptitude 1) to regulate the temperature of their home (Dactylurina, Hypotrigona) or 2) to establish themselves in a vacant section of a termitary where they take advantage of the stabilised temperature ( $T$. eburnensis). Some other bees have been able to increase their area of dispersion by the discovery of biotopes similar enough to the ones of their aboriginal (Meliponula). Finally some species have not been to escape from their narrow and specialised biotope (Meliplebeia). Apis mellifica var. adansonii is specially studied in this work because the physiological researches on the european varieties allow interesting comparisons. It is

* Ce travail a été effectué dans le cadre de la R.C.P. nº 60 du C.N.R.S. à Lamto en Côte-d'Ivoire et de l'Institut de Primatologie et d'Écologie équatoriale du C.N.R.S. à Makokou au Gabon. 
now established that the thermoregulation in the nest of the african bee is less perfect than that of its european relatives. However though provided with a poor aptitude, the african $A$ pis mellifica has succeded to colonise by means of adaptation comparable to those found in the stingless bees. These means include the use of subterranean nests.

\section{RÉSUMÉ}

Après une présentation des différents climats des régions africaines où cette étude sur la thermorégulation des abeilles sociales a été effectuée, nous donnons les résultats de mesures obtenues avec différentes espèces de Trigones Trigona sp. et Apis mellifica var. adansonii. Latr. Nous essayons ensuite de trouver des corrélations entre les capacités thermorégulatrices de chaque espèce étudiée et leur nidification ou leur écologie au Gabon et en Côte-d'Ivoire.

Brièvement nous avons observé que 1) certaines espèces sont cantonnées dans un biotope bien déterminé aux conditions climatiques restreintes et leur extension est en relation avec l'extension du biotope ( $T$. beccarii, $T$. bocandei $), 2$ ) d'autres espèces se sont adaptées à des biotopes assez différents, soit en développant une thermorégulation plus parfaite (Dactylurina, Hypotrigona), soit en modifiant la structure de leur nid (Dactylurina) ou en sachant s'adapter à un environnement dont les oscillations du microclimat ne dépassent pas leurs capacités autorégulatrices (adoption de cavités dans des termitières, T. eburnensis).

Apis mellifica var. adansonii est l'objet d'un intérêt particulier. En effet, la physiologie des différentes races européennes d'Apis mellifica est maintenant bien connue et il nous est possible, pour cette espèce, d'établir des comparaisons entre races européennes et races africaines. Cette Abeille d'Afrique a des capacités de régulation thermique assez imparfaites comparée à nos Abeilles d'Europe. Toutefois elle peut survivre dans des biotopes très variés, car elle adopte les mêmes techniques de nidification que certaines Trigones. Elle peut ainsi nidifier dans des cavités souterraines.

\section{I. - INTRODUCTION}

La thermorégulation d'Apis mellifica L. a fait l'objet d'études assez nombreuses qu'il est possible de répartir en trois chapitres, 1) la thermorégulation de l'abeille isolée ou de quelques abeilles groupées dans une ruchette, 2) la thermorégulation globale dans la ruche, 3) la thermorégulation dans la pratique apicole.

Mes conditions de travail en Afrique, dans un laboratoire de campagne, et mes préoccupations d'apidologue du moment m'ont amené à aborder le problème sous son deuxième aspect : les colonies de diverses abeilles africaines des tribus Trigonini et Apini ont-elles des possibilités de thermorégulation semblables à celles de leurs cousins d'Europe et ces possibilités donnent-elles des indications sur leur choix, sur leur technique de nidification, sur leur succès ou leur échec dans la colonisation des divers biotopes africains étudiés?

On le sait, chez Apis mellifica, la température du nid de couvain est stable quelles que soient les conditions externes de température. Avec l'humidité, cette dernière doit en effet être optimale pour assurer un développement larvaire parfait à l'intérieur des colonies. Les deux courbes ci-jointes nous présentent clairement ce phénomène dans les conditions climatiques d'Europe, où la température externe est généralement inférieure à celle du couvain, 
(BÜDEL) (fig. 1) et subtropicales où elle est habituellement supérieure (LENSKY) (fig. 2). Ces abeilles sont donc capables non seulement de réchauffer l'air à proximité du couvain mais aussi de le refroidir suivant les besoins de celui-ci.

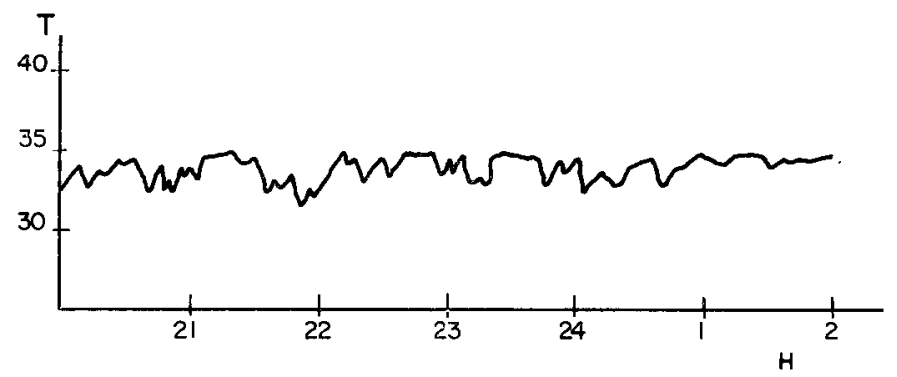

Fig. 1. - (d'après BǜEL). Courbe des températures mesurées à l'intérieur du couvain en Allemagne en plein mois de juillet.

Aвв. 1. - (nach BüDEL). Im Innern des Brutnestes gemessene Temperaturen, Deutschland, Mitte Juli.

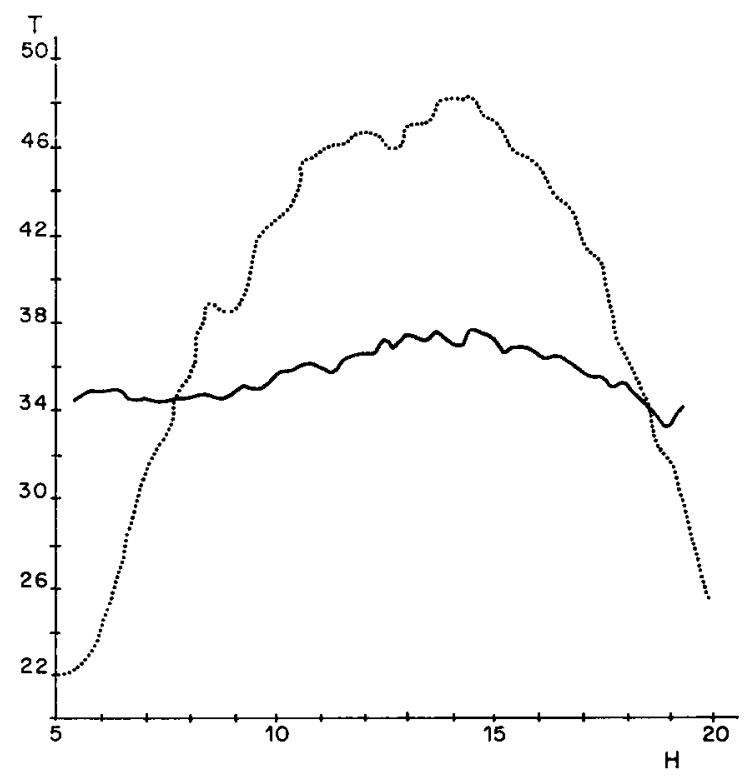

FIG. 2. - (d'après LENSKy). Variations thermiques à l'intérieur du couvain (traì plein) au cours d'une journée chaude en Israël en fonction des variations thermiques mesurées dans la serre où se trouvait la colonie. (pointillé)

ABB. 2. - (nach LeNsKy). Wärmeschwankungen im Brutnest (ausgezogene Linie) während eines warmen Tages in Israel, verglichen mit denen des Treibhauses, in dem der Bienenstock stand.

Les abeilles sociales d'Afrique ont les mêmes nécessités de thermorégulation. Cependant ces abeilles n'hibernent pas et n'interrompent pas toute activité durant une partie de l'année car, nous allons le voir, les plus basses températures ne sont pas excessives et ne durent que peu de temps. 
Or, après quelques années de séjour dans ces régions du globe et quelques déplacements à l'intérieur du continent africain, on s'aperçoit assez rapidement que certaines espèces se cantonnent dans des biotopes bien étroits et que d'autres au contraire sont adaptées à des milieux aussi différents que le sont les savanes ivoiriennes ou les forêts gabonaises. Quelles sont donc les raisons de ces expansions spécifiques si différentes?

\section{II. - PROFil DES VARIATIONS DE TEMPÉrature DANS LES DIVERS BIOTOPES OU ONT ĖTÉ EFFECTUÉES NOS OBSERVATIONS.}

A Lamto (Côte-d'Ivoire), en climat tropical, comme à Makokou (Gabon) en climat équatorial, on distingue 4 saisons. Cependant, dans la première station, la petite saison sèche ou la petite saison humide peuvent disparaître totalement durant une année ou une autre. Dans la seconde, en revanche, elles arrivent toutes généralement à la date prévue. D’autre part les saisons de ces deux stations sont décalées l'une par rapport à l'autre. A Lamto, la grande saison sèche débute en novembre et se termine en février ou mars. La grande saison des pluies la suit de mars à juillet. Puis arrivent la petite saison sèche en août et la petite saison des pluies en septembre et octobre. A Makokou, la grande saison sèche va de juin à août inclus. la grande saison des pluies commence en septembre et ne cesse que vers le 15 décembre; la petite saison sèche débute autour du 15 décembre se poursuit jusqu’à la fin février tandis que la petite saison humide dure trois mois à partir de mars (fig. 3).
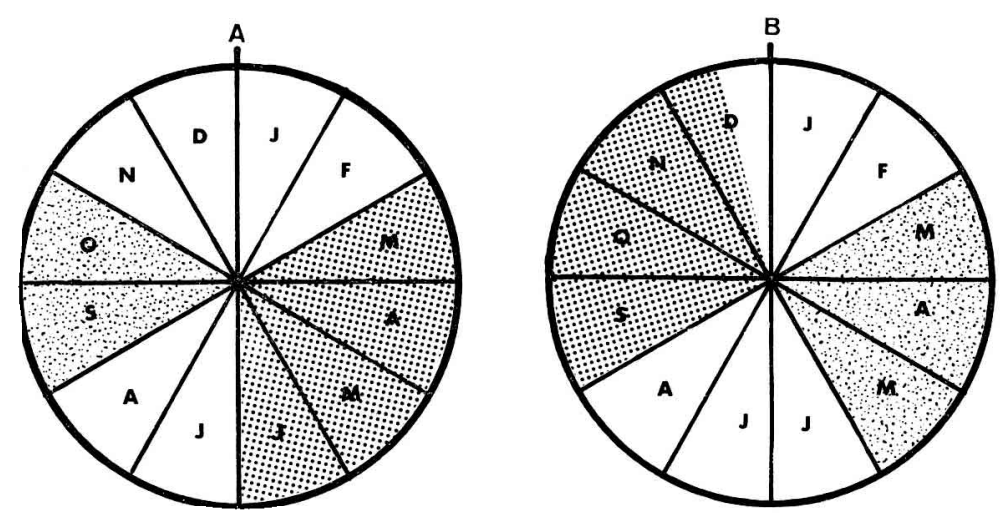

Fig. 3. - Répartition des saisons au cours de l'année à Lamto (Côte d'Ivoire) (A) et à Makokou (Gabon) (B). Le plus gros pointillé régulier représente la grande saison des pluies, le plus petit pointillé irrégulier la petite saison des pluies, le reste les saisons sèches.

Авв. 3. - Verteilung der Jahreszeiten während eines Jahres in Lamto (Elfenbeinküste) (A) und in Makolou (Gabun) (B).Grob regelmässig punktiert = grosse Regenzeit, schwach unregelmässig punktiert $=$ kurze Regenzeit, der Rest = Trockenzeiten. 


\section{1. — La savane de Lamto (Côte-d'Ivoire)}

A Lamto, la station biologique se trouve à la pointe d'une savane pénétrant comme un coin dans la forêt qui longe la côte atlantique. Elle est constituée de deux milieux typiques, la savane proprement dite et la forêt galerie qui y forme des îlots (fig. 4). Dans la savane croissent des graminées qui tapis-

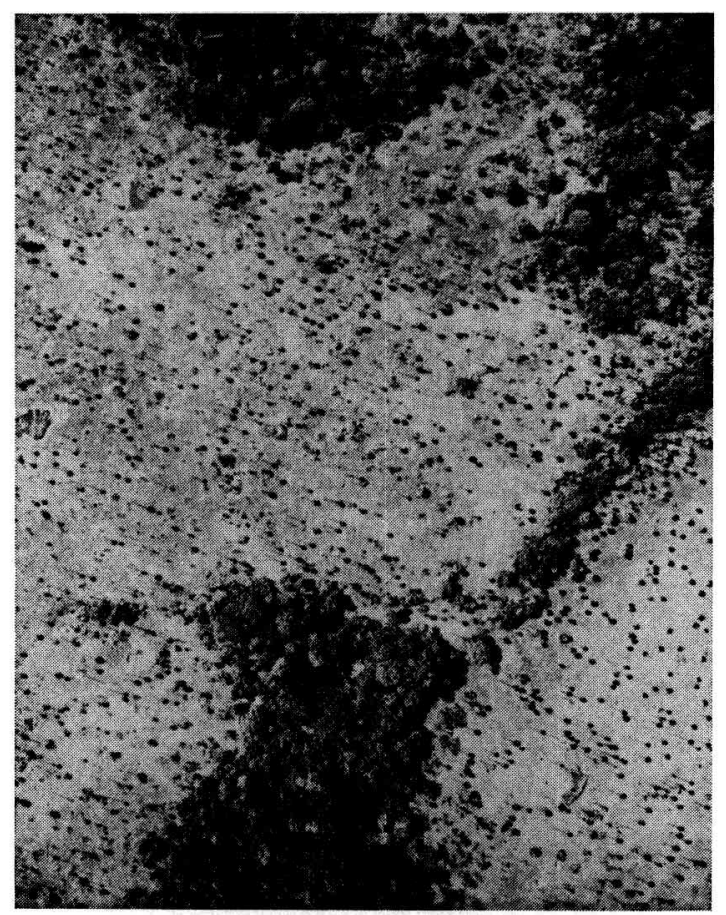

Fig. 4. - Vue aérienne de la savane de Lamto (Côte d'Ivoire). On y découvre au milieu d'unezone peu arbustive plantée de palmiers rôniers (Borassus), de Crossopterix... des forêts galeries où trouvent refuge les abeilles ayant un système de contrôle thermique peu développé (Meliponula, un certain nombre d'Axestotrigona et même d'Apis mellifica).

Aвв. 4. -- Luftbild der Savanne von Lamto (Elfenbeinküste). Man entdeckt dort inmitten eines von wenigen Sträuchern bedeckten Gebietes, das mit Palmen (Borassus) und mit Crossopterix bepflanzt ist, Galeriewälder, in denen Bienen mit schwach entwickelter Wärmekontrolle Unterschlupf finden (Meliponula, eine gewisse Anzahl von Axestotrigona und sogar Apis mell.).

sent le sol uniformément et des arbres souvent petits. La plupart d'entre eux présentent des troncs et des branches avec un large canal central dont l'origine est encore inconnue. Les abeilles et les fourmis colonisent toutes ces cavités.

La figure (5) donne la courbe moyenne des températures de huit années (1962 à 1969 inclus) d'après les relevés publiés par J.-L. Tournier dans le bulletin de Lamto de la R.C.P. 60 du C.N.R.S. Dans cette savane, on le voit 
la température est toujours élevée (moyenne 2703) et il n'y a qu'un faible écart thermique entre les différentes saisons : les moyennes quotidiennes vont de $25^{\circ}$ à $30^{\circ}$ en saison des pluies et de $24^{\circ}$ à $30^{\circ}$ en saison sèche. Cependant les maxima et les minima de température durant la première sont $20^{\circ}$ ou $23^{\circ}$ et $30^{\circ}$ ou $33^{\circ}$, et durant la seconde $23^{\circ}$ ou $25^{\circ}$ et $36^{\circ}$ ou $39^{\circ}$.

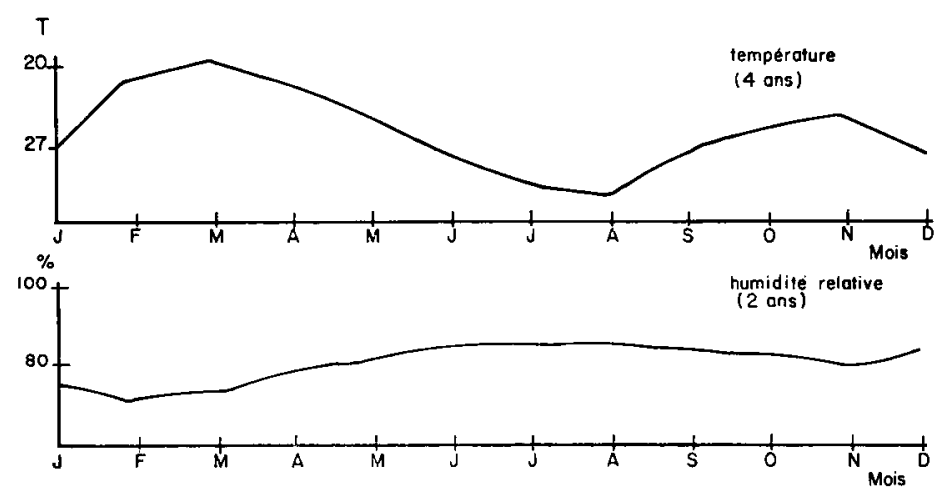

Fig. 5. - (d'après Tournier, Monnier et MÉnager). Courbes des températures et humidités relatives moyennes mensuelles sur 4 ou 2 années entre 1962 et 1966.

Abr. 5. - (nach Tournier, Monnier u. Menager). Durchschnitt der monatlichen Temperaturen und rel. Feuchtigkeit für 4 und 2 Jahre, zwischen 1962 und 1966.

\section{2. - Les forêts galeries de Lamto}

Mlle Th. MÉnager, au cours de ses mesures, a remarqué que la température moyenne de la savane et celle de la forêt galerie diffèrent en général de $2^{0}$. Durant le jour, les températures de la forêt galerie peuvent donc être assez fortes et atteindre $36^{\circ}$ en janvier. Pendant la nuit les températures des deux milieux tendent à s'égaliser mais la forêt reste un peu plus chaude (fig. 6 et 7).

Enfin l'humidité relative moyenne est $89 \%$ en forêt et $81 \%$ en savane. En forêt, elle ne descend pas au-dessous de $85 \%$. En revanche en savane et pendant la saison sèche, l'humidité peut s'établir entre $10 \%$ et $20 \%$ pendant les heures les plus chaudes alors qu'elle reste stable autour de $70 \%$ ou $80 \%$ pendant la saison des pluies.

\section{3. - L'aéroport de Makokou (Gabon)}

La figure 8 présente les courbes moyennes des températures maximales et minimales de trois années (1962 à 1964) établies par G. Dubost d'après les relevés de l'aéroport de Makokou, c'est-à-dire dans un milieu largement 


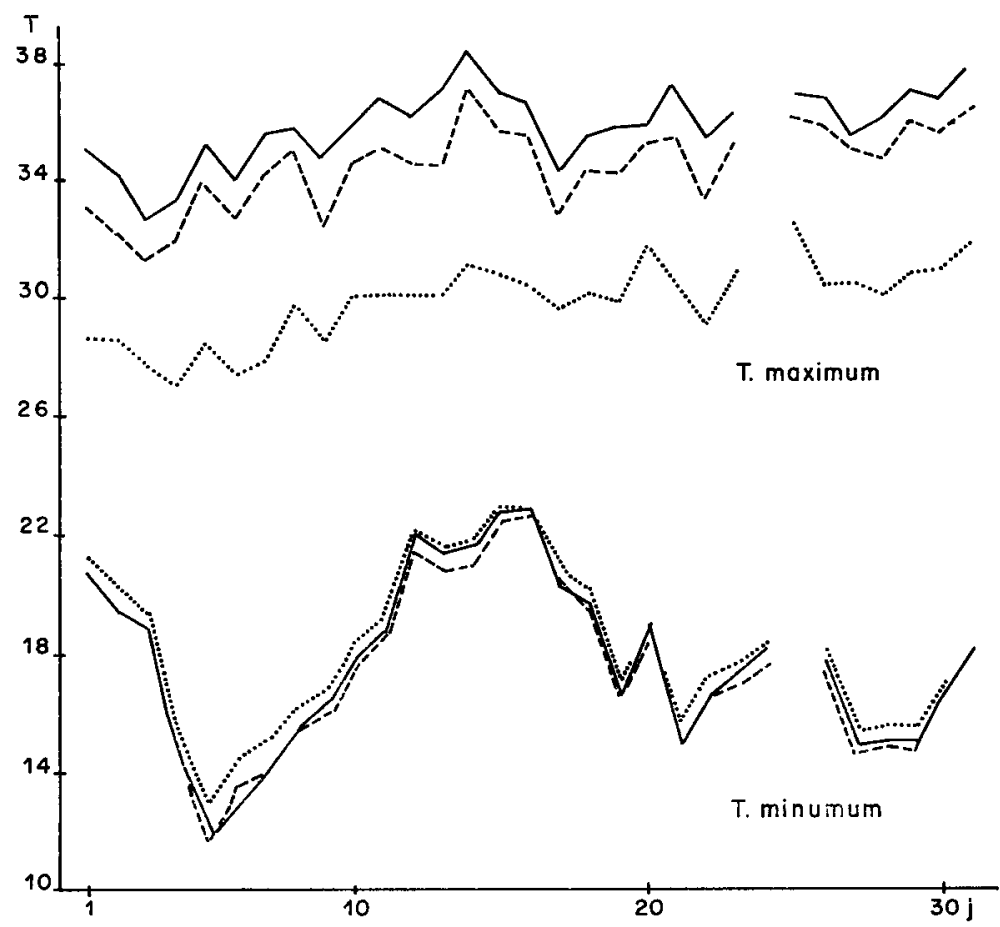

Fig. 6. - (d'après Ménager). Profil des variations thermiques durant le mois de janvier en savane (trait plein); en lisière (tirets) et en forêt galerie (pointillê). (Lamto. Janvier 1967).

Aв8. 6. - (nach MÉvager). Querschnit der thermischen Veränderungen im Januar in der Savanne (ausgezogene Linie), am Rain (gestrichelt) und im Galeriewald (punktiert). (Lamto. Januar 1967).

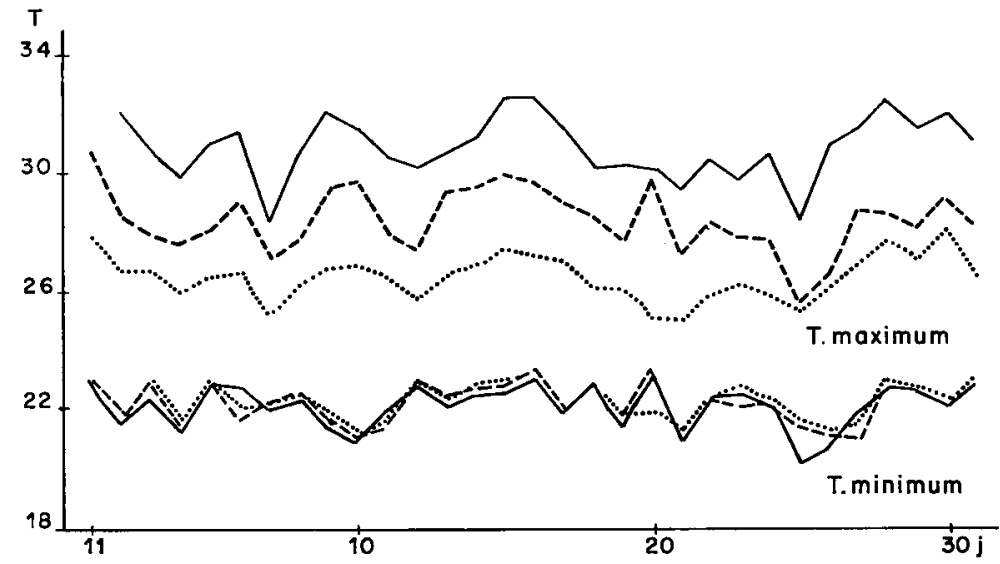

Fig. 7. - (d'après MÉNager). Courbe des variations thermiques durant le mois de juillet en savane (trait plein); en lisière (tirets) et en forêt galerie (pointillé). (Lamto. Juillet 1966).

ABb. 7. - (nach MéNager). Wärmeschwankungen im Juli in der Savanne (ausgezogene Linie), am Rain (gestrichelt) und im Galeriewald (punktiert). (Lamto. Juli 1966). 
dégagé, une sorte de clairière artificielle, dans une région forestière équatoriale. On y découvre que les températures maxima ne dépassent pas $31^{\circ}$ durant la petite saison humide et que les minima n'excèdent pas $18^{\circ}$ durant la saison sèche. En revanche, l'atmosphère est souvent saturée et l'humidité relative est toujours au-dessus de $55 \%$.
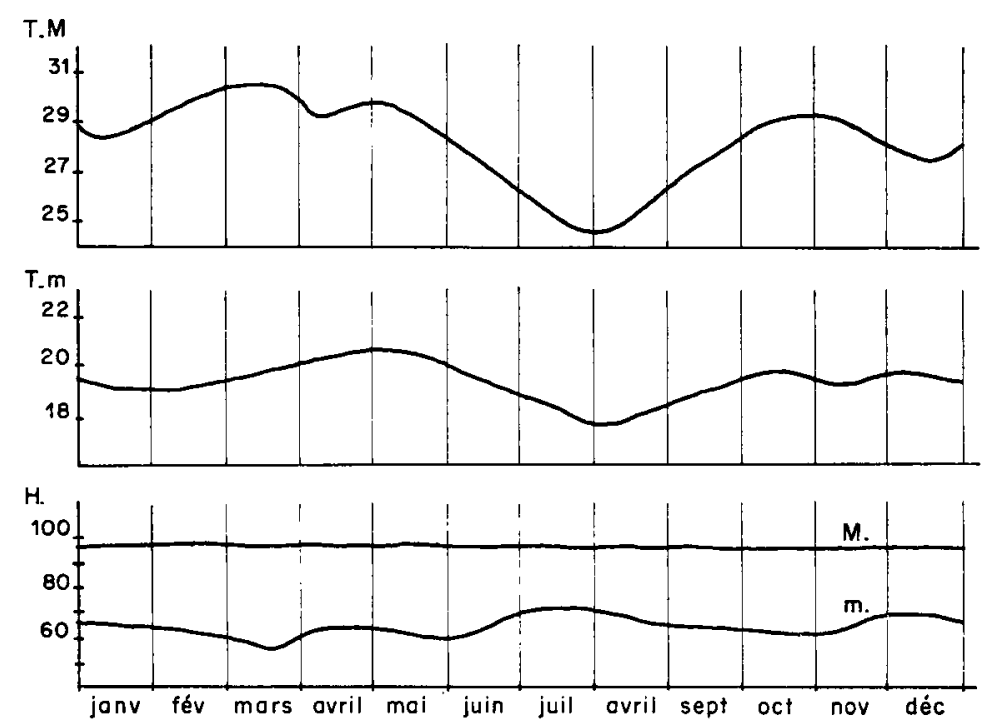

Fig. 8. - (d'après Dubost). Courbes des variations mensuelles de température et d'humidité durant 3 années (1962 à 1964) à Makokou. De haut en bas, températures maximales, températures minimales et humidité relative.

Aвв. 8. - (nach Duвost). Monatliche Schwankungen von Temperatur und Feuchtigkeit während dreier Jahre in Makokou (1962-1964). Von oben nach unten : Maxima, Minima, Feuchtigkeit.

\section{4. - La forêt équatoriale de Makokou}

Je n'ai malheureusement aucune courbe des moyennes des températures de la forêt dense à plus de 1 mètre du sol de cette station. Cependant les enregistrements que j'ai effectués durant plusieurs mois donnent une idée des phénomènes météorologiques de ce milieu particulier où vivent la plupart des abeilles gabonaises. Les résultats donnés par les divers enregistrements peuvent être résumés ainsi : les températures oscillent régulièrement entre $20^{\circ}$ et $25^{\circ}$ et l'humidité entre $80 \%$ et $95 \%$. Pendant la même période, les mêmes facteurs microclimatiques de l'aéroport ont varié entre $20^{\circ}$ et $30^{\circ}$ centigrades et entre $55 \%$ et $80 \%$ d'humidité relative.

Nous donnons ici (fig. 9) la représentation de courbes typiques du milieu forestier. 

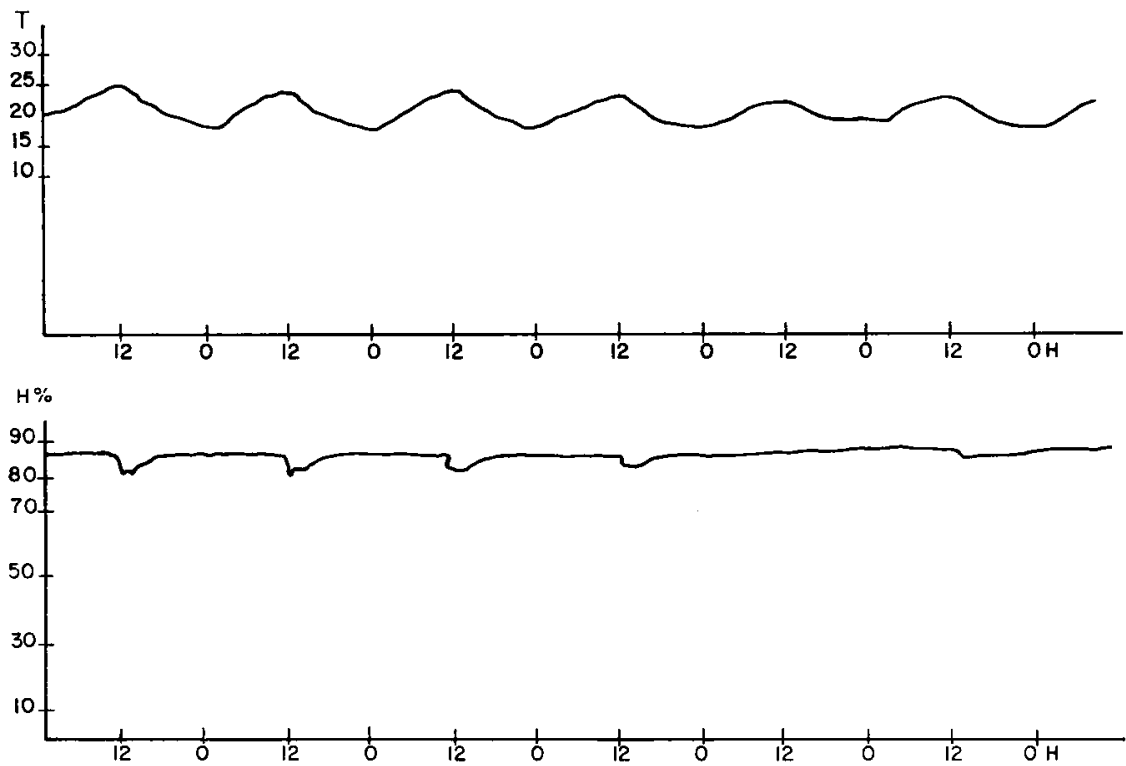

Fig. 9. - Courbes typiques des températures et de l'humidité dans la forêt secondaire entre le sol et $100 \mathrm{~cm}$ à Makokou - N'Tsibelong (Gabon).

Aвв. 9. - Typische Temperatur- und Feuchtigkeitskurven im Sekundärwald (Buschwald), am Boden und in 1 m Höhe gemessen, Makakou-N'Tsibelong (Gabun).

\section{III. - SUCCES DANS LA THERMORÉgULATION \\ CHEZ LES HYPOTRIGONES SP. ET CHEZ LES DACTYLURINES (TRIGONA STAUDINGERI).}

\section{1. _ Les Hypotrigones}

Elles font partie des plus petites abeilles sociales d'Afrique. Elles sont réparties dans la plus grande partie de l'Afrique depuis le sud du Sahara et à Madagascar.

Le groupe est d'ailleurs très ancien puisque récemment deux exemplaires fossilisés ont été trouvés dans l'ambre de la Baltique. C'est une Hypotrigone très voisine d'Hypotrigona araujoi Mic. Les abeilles de ce genre ont donc peu évolué depuis des millions d'années.

Les insectes de ce groupe sont d'une détermination très délicate sinon impossible car ils ne diffèrent en général les uns des autres que par leurs dimensions. C'est dire qu'ils sont très semblables et c'est ce qui justifie une étude globale du groupe. Enfin, à ce sujet, il est bon de rappeler que, par des nourrissements spéciaux, j'ai pu obtenir des animaux plus petits que ceux qui habitent dans la même colonie. 
Or, on trouve ces insectes dans des biotopes aussi divers que la savane et la forêt sous des climats aussi différents que le climat tropical (Lamto) ou équatorial (Makokou) donc aussi bien dans les forêts congolaises que dans les savanes soudanaises et guinéennes.

Les colonies de ces minuscules abeilles sont assez peu populeuses (quelques centaines d'individus) et elles ont des facilités extraordinaires d'adaptation aux divers biotopes et lieux de nidification. Leur habitat normal est le tronc ou la branche d'un arbre creux, mais elles s'installent facilement dans les endroits les plus insolites, par exemple à l'intérieur d'une serrure, dans un tube conducteur de fils électriques, dans des cavités creusées dans d'anciennes termitières. $J$ 'en ai trouvé aussi dans un tube en $U$ entourant le fourgon d'une camionnette 2 CV. Citroën! A l'époque où les Gabonais revêtaient leurs toits de feuilles de palmier ces abeilles venaient se loger dans l'épaisseur du feuillage (fig. 10), ou

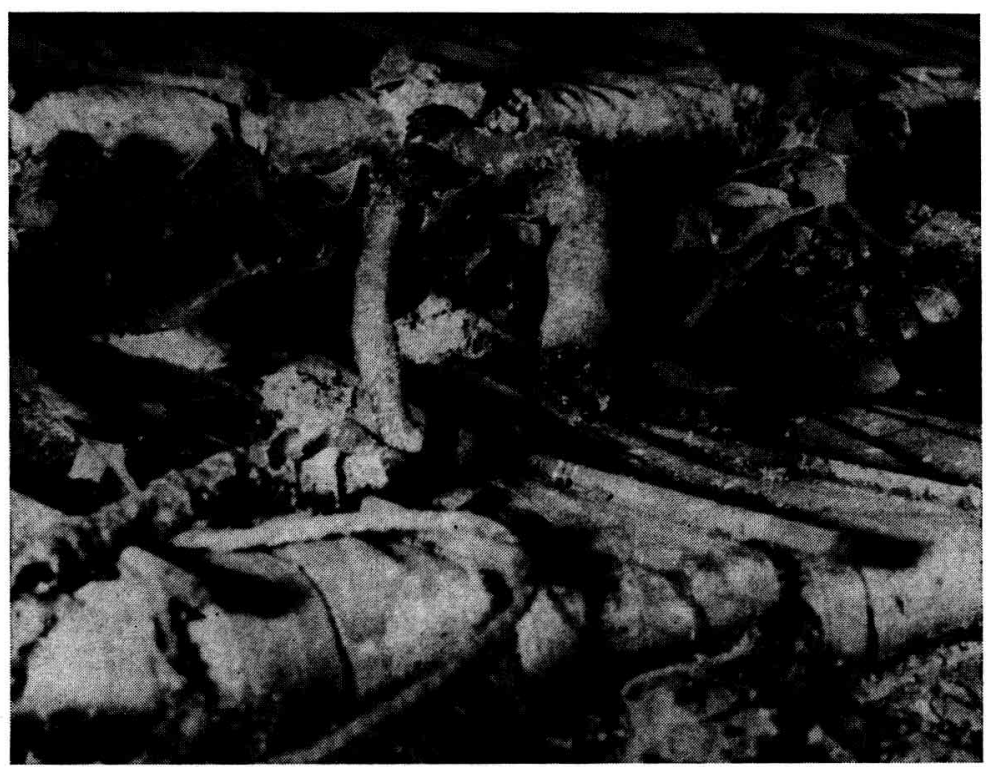

FIG. 10. - Nids d'Hypotrigones ayant élu domicile dans les feuilles de palmier qui recouvrent le toit des cases (Makokou).

Aвв. 10. - Nester von"Hypotrigonen in Palmblättern, mit denen die Hausdächer gedeckt sind.

bien encore dans la partie creuse des bambous qui servaient de charpente aux maisons indigènes. Or, en ces lieux, les colonies sont soumises à de très grandes variations thermiques auxquelles les abeilles sont parfaitement adaptées.

La structure du nid de ce groupe d'abeilles est extrêmement simple : d'une extrémité à une autre de l'habitation on découvre des amas de cellules de réserve, des amas de cellules de couvain sous enveloppe de cire et enfin, près 
de la sortie, s'ouvre un tube de cire qui serpente longuement à l'intérieur de l'habitacle et se prolonge à l'extérieur sur plusieurs centimètres pour laisser aux butineuses la possibilité d'entrer et de sortir. Sur le bord externe du tube se tiennent les gardiennes et, derrière elles pendant les fortes chaleurs, de nombreuses ventileuses à la queue leu leu, la tête dirigée vers le substrat et vers l'intérieur de la ruche, les pattes antérieures abaissées, les pattes moyennes, postérieures et l'abdomen soulevés en direction de la sortie. Le bourdonnement est intense.

\section{2. - Les Dactylurines}

Contrairement à la précédente espèce qui aménage des cavités, celle-ci construit ses nids de toutes pièces. Le genre Dactylurina est d'ailleurs le seul en Afrique à nicher ainsi. J'ai vu ses nids pour la première fois dans les forêts secondaires gabonaises (fig. 11).

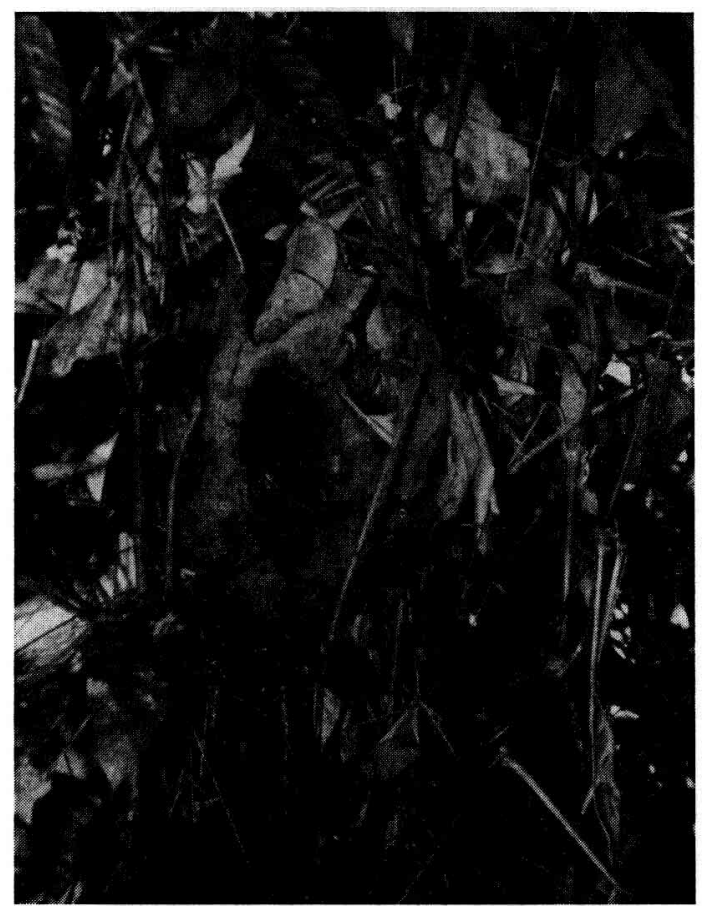

Fig. 11. - Nid de Dactylurina dans la forêt secondaire (Makokou).

Aв8. 11. - Nest von Dactylurina im Sekundärwald (Makokou).

Ils ont grossièrement la forme d'une boule et se trouvent en général à peu près à deux mètres de hauteur, très bien protégés par une épaisse couche de feuilles sous laquelle les abeilles pénètrent pour trouver leur entrée à la base 
de l'édifice. La longueur moyenne de ces nids est en général $30 \mathrm{~cm}$ et leur largeur 25. La cavité réservée au couvain mesure environ une quinzaine de centimètres.

De l'extérieur à l'intérieur, le nid comprend 1) une fine pellicule de résine d'un demi-millimètre d'épaisseur toujours bien entretenue par les bâtisseuses : les réparations des brèches sont effectuées dans les heures qui suivent la lésion, 2) des couches plus ou moins concentriques, anastomosées et perforées de résine dure et cassante d'une épaisseur de $9 \mathrm{~cm}$ en moyenne, 3) les cellules de couvain et des réserves. L'entrée du nid est située face au sol. Les ouvrières entrent et sortent par 1 ou 2 ouvertures d'environ $7 \mathrm{~cm}$ de diamètre (fig. 12).

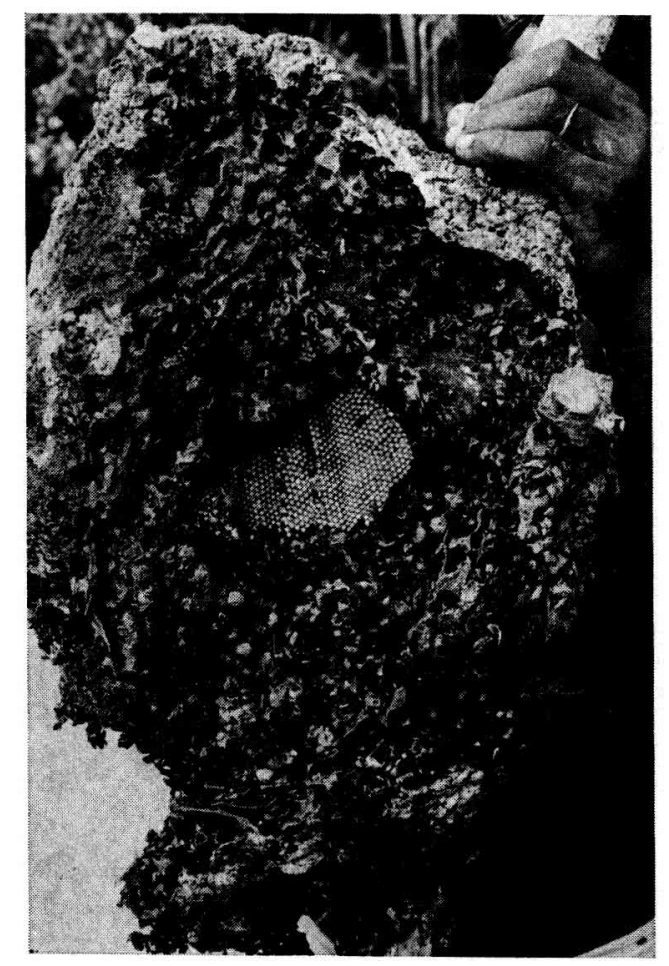

FIG. 12. - Coupe d'un nid de Dactylurina de la savane (Côte d'Ivoire) à 2 mètres du sol. Aв8. 12. - Schnitt durch ein Dactylurina - Nest in der Savanne (Elfenbeinkïste), 2 m über der Erde.

L'étude de l'hygrorégulation et surtout de la thermorégulation des nids de Trigones au Gabon révèle des phénomènes curieux. L'humidité et la température dans les plantations où l'on trouve souvent Dactylurina staudingeri sont, nous l'avons vu, relativement stables. Les nids de cette espèce sont cependant soumis à des variations thermiques suffisantes pour déclencher tout un comportement intéressant à étudier. Comment donc ces Trigones refroidissent-elles 
leur nid ? 10) elles ouvrent d'abord un gros orifice semblable à celui qui leur sert d'entrée sur le côté opposé à ce dernier à la base du nid, 20) vers le haut elles percent des petits trous plus ou moins nombreux à la surface de l'enveloppe du nid, $3^{\circ}$ ) elles ventilent.

Examinons maintenant en détail chacune des techniques utilisées par ces abeilles pour climatiser leur habitation.

10) La présence d'un second gros orifice est assez constante à la base des nids mais, tandis que le premier conserve sa taille égale durant plusieurs jours, le second varie d'un jour à l'autre et peut être totalement obstrué.

$2^{\circ}$ ) Des ouvrières travaillent à l'intérieur du nid dans la partie supérieure de l'enveloppe, creusent des orifices d'un millimètre de diamètre environ. Leur nombre par $\mathrm{cm}^{2}$ et leur répartition à la surface du nid varient au cours de la journée. Ne disposant à cette époque que d'un matériel assez simple, je n'ai établi une corrélation qu'entre le nombre des orifices et la température ambiante des nids répartis dans une plantation de cacaoyers.

Nous avions percé des trous carrés de différentes tailles dans des feuilles de papier souple afin de pouvoir les manipuler aisément et les appliquer facilement à la surface arrondie mais irrégulière du nid d'abeille. Il s'est avéré par la suite que les aires délimitées par les bords des orifices n'étaient pas exactement dans les rapports, $1 / 5,1 / 20,1 / 40$, comme nous le désirions. C'est pourquoi dans le tableau 1 , le chiffre (1) représente une surface de $16 \mathrm{~cm}^{2}$, (2) une de $113 \mathrm{~cm}^{2}$, (3) une de $380 \mathrm{~cm}^{2}$ et (4) une de $700 \mathrm{~cm}^{2}$. Les résultats sont faciles à apprécier : les abeilles multiplient les orifices dans la pellicule qui recouvre le nid d'autant plus que la température est élevée, en revanche, elles le bouchent lorsqu'arrive la fraîcheur des nuits de Makokou qui est à une altitude de 600 mètres.

On peut rapprocher ce comportement de Dactylurina staudingeri de celui qui a été signalé par Nogueira-Neto dans les nids de certaines Mélipones sudaméricaines où une partie de la bâtisse baptisée « batumen » est percée de minuscules orifices qui permettent grâce à la ventilation des abeilles de climatiser l'atmosphère intérieure.

30) A l'époque de nos recherches, nous ne possédions aucun instrument pour mesurer l'intensité du bruit produit par les ventileuses au cours de la journée à l'intérieur du nid. Cependant il ne fait aucun doute que le bruit produit par les ventileuses va en s'amplifiant lorsque le soleil réchauffe fortement l'atmosphère : presque nul le matin ou au moment des pluies, il est très audible à quelques centimètres du nid au début de l'après-midi. Les ventileuses peu nombreuses à l'entrée vers le bas, se trouvent réparties par conséquent dans. tout le dôme labyrinthoïde du nid. 
TABL. I. - Thermo et hygrorégulation par l'ouverture

TABELle 1. - Wärme-und Feuchtigkeitsregulierung durch Offnen

\begin{tabular}{|c|c|c|c|c|}
\hline $\begin{array}{c}\begin{array}{c}\text { Heure } \\
\text { des } \\
\text { mesures }\end{array} \\
\text { Zeit d. } \\
\text { Messungen }\end{array}$ & $\begin{array}{c}\text { Température } \\
\text { ambiante } \\
\text { Umgebungs- } \\
\text { Temperatur }\end{array}$ & $\begin{array}{c}\text { No référ. } \\
\text { de la } \\
\text { colonie } \\
\text { Nr. d. } \\
\text { Volkes }\end{array}$ & 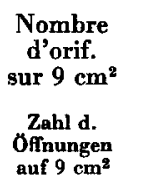 & $\begin{array}{c}\text { Surf. cou- } \\
\text { verte par } \\
\text { les orif. } \\
\text { Oberfläche } \\
\text { mit Offnungen } \\
\text { bedeckt }\end{array}$ \\
\hline \multirow[t]{7}{*}{09,00} & 23 & 1 & 4 & 3 \\
\hline & & 6 & 0 & 0 \\
\hline & & 7 & 22 & 2 \\
\hline & & 8 & 7 & 3 \\
\hline & & 9 & 2 & \\
\hline & & 12 & 0 & 0 \\
\hline & & 5 & 2 & 3 \\
\hline \multirow[t]{9}{*}{15,00} & 27 & 1 & 7 & 3 \\
\hline & & 6 & 1 & 2 \\
\hline & & 7 & 21 & 3 \\
\hline & & 8 & 17 & 3 \\
\hline & & 9 & 3 & 2 \\
\hline & & 12 & $\mathbf{3}$ & 2 \\
\hline & & 13 & 30 & 3 \\
\hline & & 5 & 6 & 2 \\
\hline & & 3 & 21 & 4 \\
\hline \multirow[t]{7}{*}{10,30} & 26 & 1 & 8 & 3 \\
\hline & & 6 & 1 & \\
\hline & & 7 & 24 & 3 \\
\hline & & 8 & 18 & 3 \\
\hline & & 9 & 6 & 2 \\
\hline & & 5 & 4 & 3 \\
\hline & & 3 & 27 & 4 \\
\hline \multirow[t]{7}{*}{15,30} & 28 & 1 & 10 & 3 \\
\hline & & 6 & 1 & \\
\hline & & 7 & 24 & 3 \\
\hline & & 8 & 20 & 3 \\
\hline & & 9 & 6 & 2 \\
\hline & & 5 & 5 & 4 \\
\hline & & 3 & 28 & 4 \\
\hline \multirow[t]{8}{*}{08,40} & 23 & 1 & 5 & 1 \\
\hline & & 6 & o & \\
\hline & & 7 & 9 & 2 \\
\hline & & 8 & 10 & 3 \\
\hline & & 9 & 0 & \\
\hline & & 5 & 5 & 3 \\
\hline & & 3 & 18 & 3 \\
\hline & & 13 & 3 & 2 \\
\hline
\end{tabular}


et la fermeture d'orifices dans l'enveloppe de résine du nid

und Schliessen der Lufilöcher in der Harzhülle des Nestes

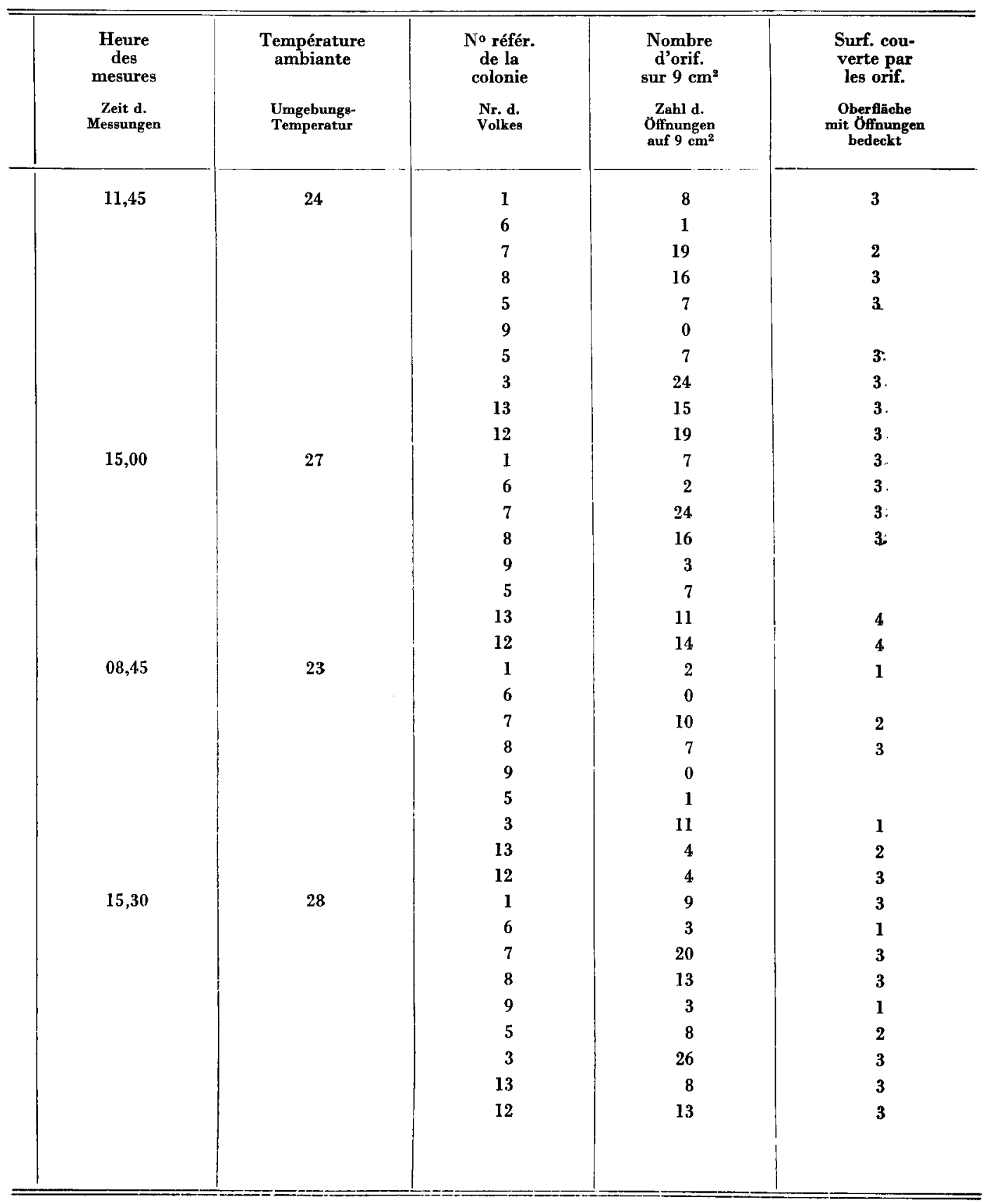


C'est alors que m'a été donné l'occasion de travailler dans les savanes de Côte-d'Ivoire et de découvrir des Dactylurines dans un biotope très différent de celui que je connaissais jusqu'alors. Les nids dans ce cas ont été rencontrés à des hauteurs insoupçonnées, de 3 à 20 mètres aisément (fig. 13). Ils étaient en

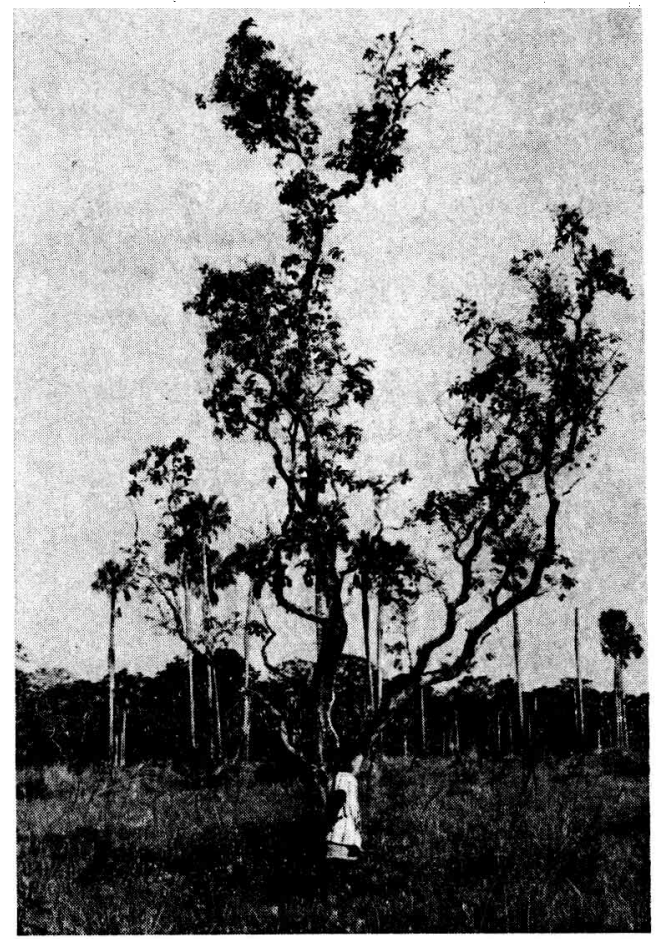

Fig. 13. - Nid de Dactylurina dans la savane de Lamto. Aвв. 13. - Nest von Dactylurina in der Savanne von Lamto.

général à peine protégés par un feuillage peu dense parfois même sans protection du tout, accolés au tronc des arbres (palmier par exemple). La thermorégulation, décrite précédemment, doit être alors très efficace pour permettre aux colonies d'abeilles de survivre aux fortes chaleurs caniculaires de certaines saisons de l'année.

Les nids de Dactylurina staudingeri de Côte-d'Ivoire présentent d'ailleurs quelques particularités originales qui sont peut-être d'ailleurs en corrélation avec le problème qui nous intéresse. En effet, si les dimensions de la cavité contenant le couvain restent à peu près les mêmes, celles du nid proprement dit sont beaucoup plus grandes; la largeur passe de 25 à $27 \mathrm{~cm}$ et la longueur de 30 à $4,1 \mathrm{~cm}$. C'est dire que la coquille de résine dure et labyrinthoüde devient plus épaisse et par là beaucoup plus isolante par la présence de plus nombreuses cavités remplies d'air. 


\section{3. - Le cas de Meliponula bocandei}

Cette grosse espèce est ubiquiste et signalée dans diverses régions africaines, l'Angola, l'Ouguanda, le Gabon, la Côte-d'Ivoire. A-t-elle su aussi s'adapter aux conditions climatiques des divers biotopes que nous connaissons par une thermorégulation efficace comme l'espèce précédente ? En fait, Meliponula est une espèce essentiellement forestière. Elle butine dans la savane, elle pille la nourriture sucrée donnée à des animaux encagés de la station, mais elle gîte dans les forêts galeries qui découpent la savane à rôniers de Lamto. Ses nids semblables à ceux du Gabon, sont bien cachés à l'intérieur d'épais troncs d'arbres de la forêt. Cette abeille largement répandue en Afrique a donc su simplement nidifier dans des biotopes similaires quelle que soit la région adoptée. En revanche les abeilles du genre Meliplebeia (type : Trigona beccarii) n'ont pu s'évader des forêts épaisses et humides (voir les enregistrements de la forêt de Makokou où elles trouvent à nidifier à une centaine de centimètres dans des sols humides ou même détrempés) (fig. 14).

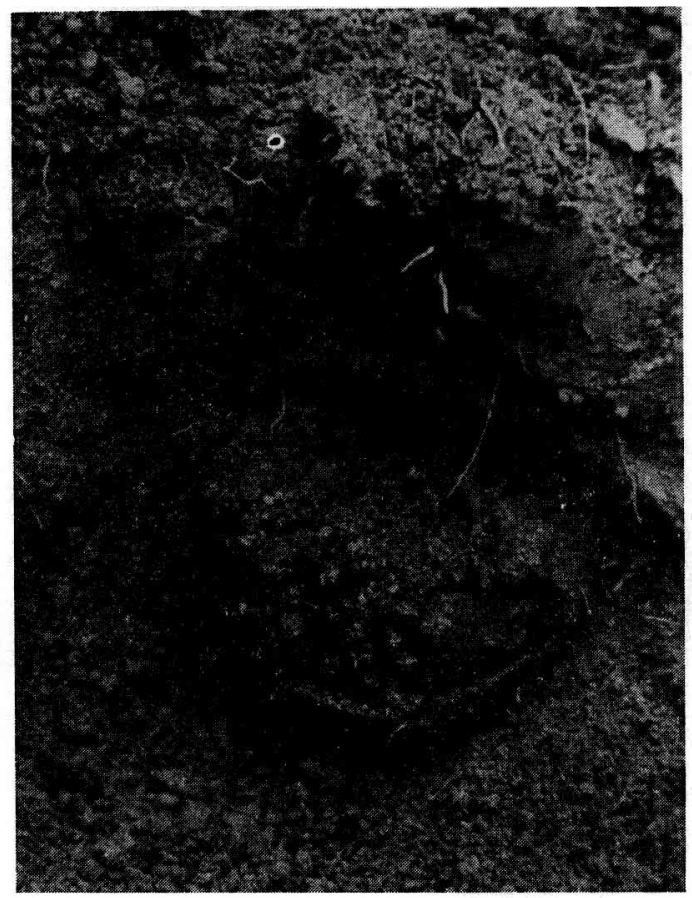

Fig. 14. - Nid de Trigona (Meliplebeia) beccarii à 1 mètre de la surface du sol dans un terrain sablonneux et humide de Makokou.

Aвв. 14. - Nest von Trigona (Meliplebeia) beccarii, $l \mathbf{m}$ über dem Boden in sandigem, feuchtem Gebiet von Makakou. 


\section{IV. - LES RÉgULATIONS IMPARFAITES DE LA TEMPERATURE CHEZ TRIGONA (APOTRIGONA) NEBULATA. ET CHEZ TRIGONA (AXESTOTRIGONA) EBURNENSIS.}

\section{1. - Trigona (Apotrigona) nebulata}

Cette espèce est très commune à Makokou. Elle est, semble-t-il, strictement inféodée à des Nasutitermes arboricoles dans la forêt secondaire (fig. 15). Quatre espèces d'oiseaux creusent ces nids de Termites de 30 à $50 \mathrm{~cm}$ de diamètre, y logent et y couvent leurs œufs. Les jeunes partis, la cavité devient

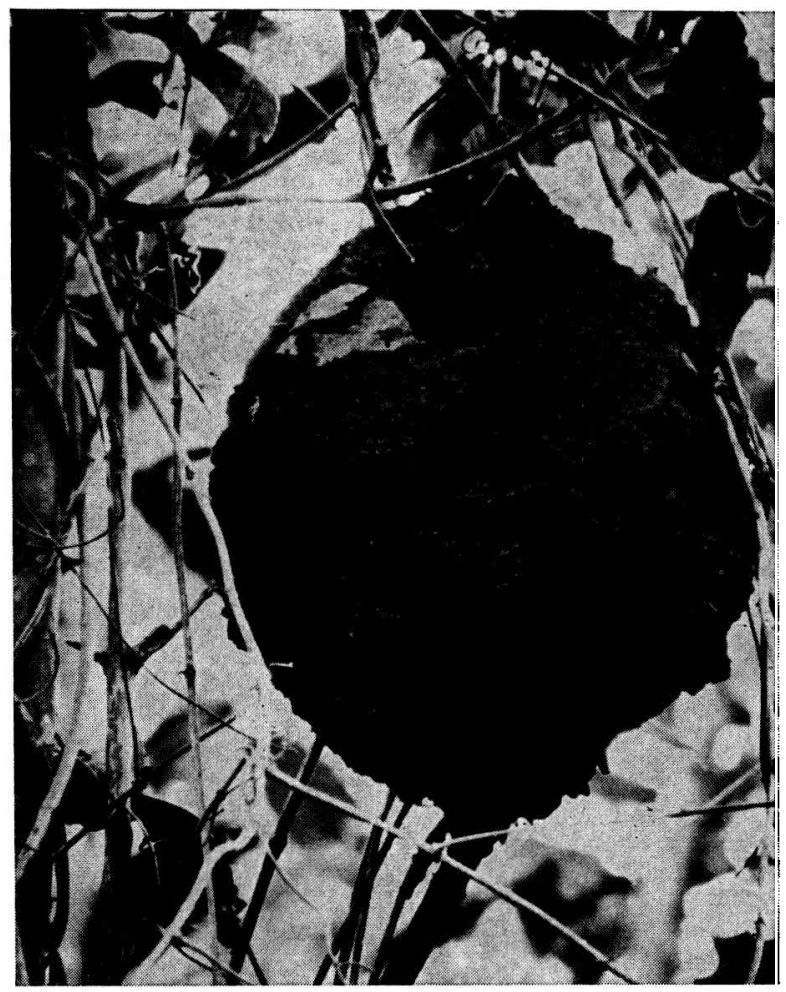

FIg. 15. - Nid de Trigona (Apotrigona) nebulata dans une termitière de Nasutitermes. (Photo A. R. Devez).

Aв8. 15. - Nest von Trigona (Apotrigona) nebulata in einem Termitenhiigel von Nasutitermes (Foto A. R. DEVEz).

libre et peut servir d'habitation aux abeilles qui s'isolent des Termites en déposant une coque épaisse de résine sur les parois intérieures de la cavité. Les termites restants et les abeilles cohabitent donc ainsi.

La description de la structure du nid a peu d'intérêt pour le sujet de ce 
mémoire. En revanche la structure du nid du termite rappelle assez celle du nid de Dactylurina. En effet, si le matériau utilisé par les Nasutitermes est différent, des sécrétions stercorales, de la terre et des débris végétaux au lieu de résine et de cire, la texture labyrinthoïde du nid est la même : de nombreuses et fines cavités donnent au ciment du nid l'aspect d'une éponge sèche.

A part le logement, cette abeille trouve-t-elle d'autres avantages à habiter ce type de nid de Termite? Et si ces avantages existent, ne sont-ce pas les mêmes qui poussent d'autres abeilles sociales à adopter soit des nids de Termites d'autres espèces, soit des nids de Fourmis. En effet, ces cas ne sont pas exceptionnels puisqu'en Amérique du Sud Trigona latitarsi et Trigona kohli colonisent l'habitat d'Eutermes rippertii. Trigona cupira, Trigona fulviventris et Trigona pallida connaissent aussi les qualités des nids de Nasutitermes, les recherchent et y établissent leur domicile.

J'ai donc introduit des colonies dans des boîtes en bois mises à l'abri sous un toit afin de mesurer les variations thermiques des boîtes colonisées et des boîtes vides au moyen des sondes d'un thermomètre enregistreur. L'une d'entre elles était fixée dans le couvain ou dans la ruche vide, l'autre sous le toit de la ruchette d'élevage.

Les résultats des enregistrements conduisent tous à la même conclusion : la thermorégulation, effectuée par les colonies de Trigona nebulata, est très imparfaite sinon inexistante (fig. 16). Pourtant un bruit de ventilation est
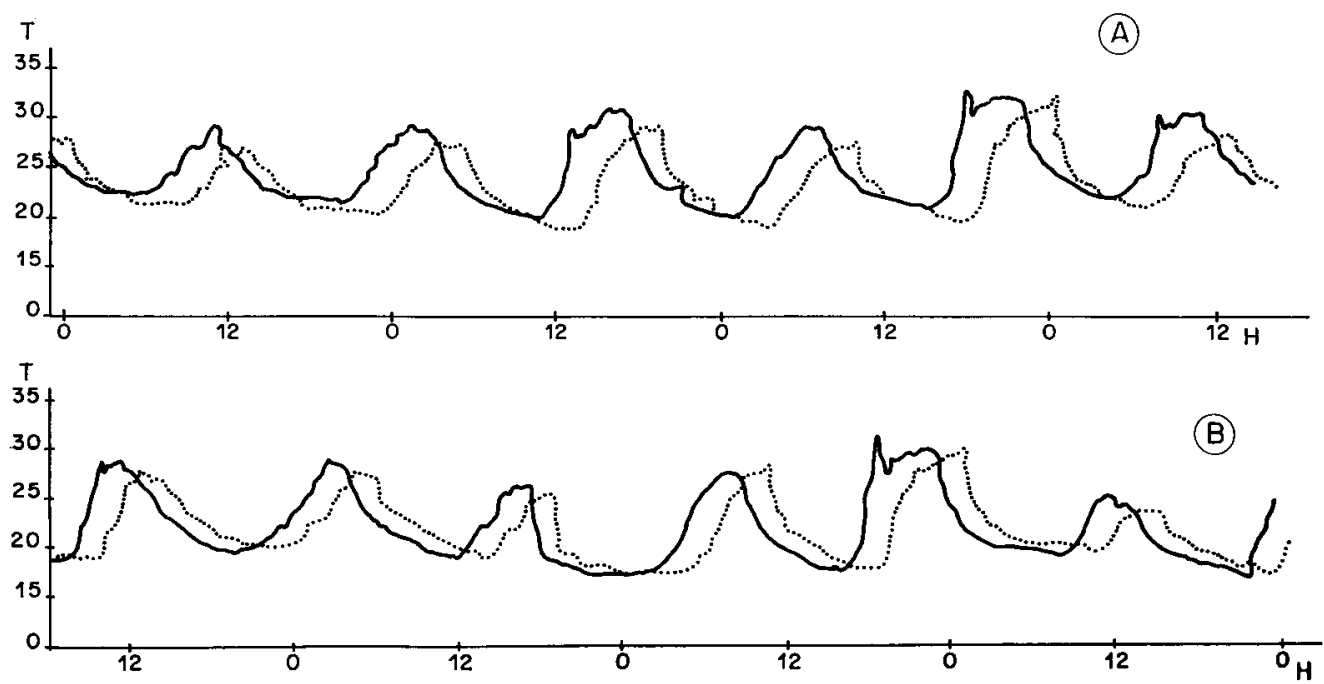

FIG. 16. - (A) Profil des variations thermiques à l'intêrieur du nid d'une ruchette contenant une colonie de Trigona (Apotrigona) nebulata (trait plein) et à l'air, air libre (pointillé). (B) Courbe des températures mesurées sous abri à l'intérieur d'une ruchette vide semblable à la précédente (trait plein), et dans l'abri (pointillé).

Aв8. 16. - (A) Wärmeschwankungen im Brutnest eines Trigona (Apotrigona) nebulata- Völkchens in einer kleinen Beute (ausgezogene Linie) und der Luft im Freien (punktierte Linie). (B) Temperaturen im Innern einer kleinen, leeren, der vorigen ähnlichen Beute, die in einem Schutzhäuschen steht (ausgezogene Linie)und im Schutzhüuschen (punktierte Linie). 
sans cesse perçu à travers les parois des ruches naturelles ou artificielles. S'agit-il d'une aération par ventilation, ou d'un moyen de déshydratation des nids? Je n'ai jamais vu de ventileuses à l'intérieur des ruches vitrées. Sontelles, comme chez les Hypotrigones, uniquement confinées dans le long et obscur tunnel de sortie? Notons enfin qu'une ruchette populeuse, fixée sur un piquet dans une clairière et sous abri, est morte en quelques semaines après un développement initial très rapide.

En résumé, les nids de Nasutitermes accrochés aux branches d'arbre à l'intérieur d'une forêt très épaisse sont abrités contre les intempéries et les rayons ardents du soleil africain. Trigona nebulata y rencontre-t-elle un site idéal pour nidifier, y trouve-t-elle comme d'autres abeilles une température assez régulière qui compense sa carence de thermorégulation?

\section{2. - Trigona (Axestotrigona) eburnensis.}

La réponse à ces questions nous a, semble-t-il, été donnée à la suite de nos propres observations et des mesures effectuées par G. Josens dans la savane de Lamto.

Une nouvelle espèce, Trigona eburnensis, nidifie en effet en pleine savane au milieu des graminées dans des nids totalement abandonnés de Bellicositermes ou de Trinervitermes trinervius (fig. 17). La plupart des cavités dans lesquelles s'installent les abeilles ont été souvent le fait de vertébrés qui viennent dégrader les nids pour se nourrir ou pour y loger. Les abeilles y élisent done domicile, à l'instar de Termites d'autres espèces ou de Fourmis. On peut ainsi trouver dans le même nid abandonné par les gros Termites plusieurs espèces de Termites, tels que Amitermes, Ancistrotermes, Pseudacanthotermes Trinervitermes et, en même temps, plusieurs espèces de Fourmis, tels Crematogaster, Camponotus, Monomorium et Odontomachus. Les nids d'Abeille sont généralement situés au cœur de la termitière. Les butineuses y entrent et en sortent par un long tunnel de résine.

Josens installa plusieurs sondes à divers endroits à l'intérieur d'un nid partiellement hypogé d'une espèce très voisine de celle qui sert d'hôte aux abeilles, Trinervitermes geminatus $w$. Ses sondes furent enfouies dans le sommet de la termitière, puis à $20 \mathrm{~cm}$ au-dessus du sol et à $40 \mathrm{~cm}$ au-dessus de la surface de la terre. Il nota alors des fluctuations thermiques liées aux variations climatiques externes (fig. 18). Elles étaient fortes dans le dôme du nid et s'atténuaient rapidement en descendant vers la terre. Il n'était pas rare de relever des températures dépassant $50^{\circ} \mathrm{C}$ dans la partie supérieure de la termitière. Pendant ce temps, les variations thermiques à l'intérieur de la termitière étaient de l'ordre de $2^{\circ}$ à $4^{0}$, la température oscillant à ce niveau autour de $30^{\circ}$. Dans cette région du nid, il n'y a pas de variations thermiques brutales. Or ces 


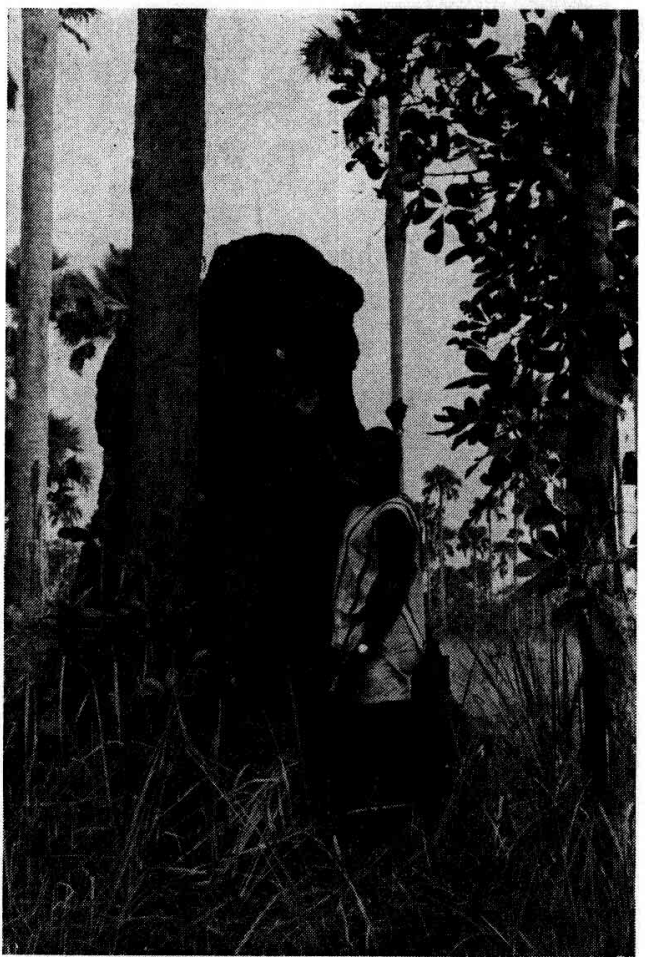

FIG. 17. - Nid abandonné de termites du Natal et réoccupé par des colonies de termites d'autres espèces, de fourmis et des abeilles sociales, Trigona (Axestotrigona) eburnensis.

Aвв. 17. - Verlassenes Termitennest in Natal, das von Kolonien mehrerer Termitenarten, von Ameisen und sozialen Bienen : Trigona (Axestotrigona) eburnensis wiederbesetzt ist.

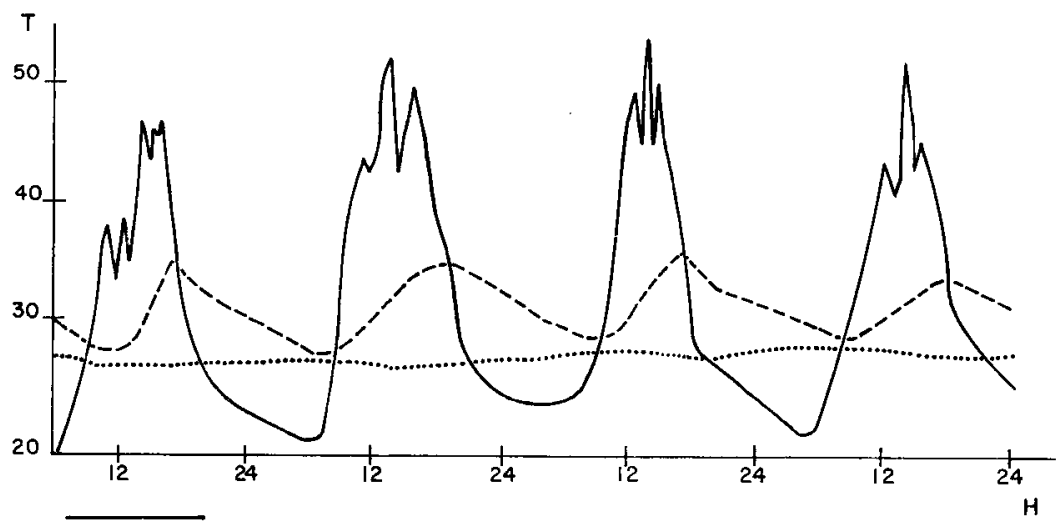

FIg. 18. - (d'après Josens). Températures mesurées sur 24 heures dans un nid épigée du termite Trinervitermes geminatus dans la savane de Lamto près du sommet (trait plein), au centre (tirets) et dans une loge souterraine (pointillê).

Aв8. 18. - (nach Josens). Während 24 Stunden gemessene Temperaturen in einem teilweise unterirdisch angelegten Nest der Termite Trinervitermes geminatus in der Savanne von Lamto; ausgezogene Linie $=$ nahe der Spitze gemessen, gestrichelt $=$ in der Mitte gemessen, punktiert $=$ in einer unterirdischen Kammer gemessen. 
températures et ces variations relevées dans la termitière sont équivalentes à celles enregistrées à une même profondeur dans la terre environnante. Notons au passage que les feux de brousse périodiques n'ont qu'une in fluence négligeable sur la température intérieure de la termitière.

Comme on pouvait le prévoir, l'auteur précédent conclut que les termites ne régulent pas la température de leur habitation mais que la structure alvéolaire de leur nid est telle qu'il en résulte une certaine inertie thermique.

On comprend donc pourquoi les abeilles tendent de profiter au maximum de ces conditions climatiques particulières qui règnent à l'intérieur des nids de termites et des nids de Fourmis. Remarquons en outre que les termitières ou les fourmilières occupées par des colonies vivantes restent toujours en parfait état et, par conséquent, que les abeilles ont un gîte qui jouit d'une efficace protection contre les intempéries si violentes dans les régions équatoriales ou tropicales.

Ainsi à côté d'espèces sociales ayant résolu assez bien le contrôle des conditions physiques de vie à l'intérieur de leur nid (Hypotrigones, Dactylurines), on rencontre d'autres espèces qui savent compenser leur carence en ce domaine en adoptant des milieux où leur existence est possible dans un macrobiotope, la forêt (Meliponula), ou dans un microbiotope, les nids d'autres espèces d'insectes (Trigona eburnensis, Trigona nebulata, etc.) où la température et l'humidité sont régulées automatiquement grâce à l'architecture du lieu d'hébergement. Ces espèces gardent ainsi des possibilités de colonisation du continent, bien que, en réalité, il leur manque la chaîne des comportements susceptibles d'assurer leur service en milieu hostile. Elles pallient ce manque en usant de stratagèmes quant aux choix des lieux de nidification.

\section{V. - UN CAS SPÉCIAL - LA THERMORÉgULATION CHEZ APIS MELLIFICA VAR. ADANSONII.}

\section{1. - La thermorégulation chez Apis mellifica var. adansonii}

A Makokou, j'ai élevé deux colonies de cette espèce dans des ruches Dadant surmontées d'une hausse vide ou très peu remplie de miel. L'une d'entre elle (ruche A) était exposée en plein soleil dans un champ de graminées; l'autre (ruche B) se trouvait dans une bananeraie où les feuilles étaient largement épanouies couvrant la ruche de leur ombre.

J'ai donc enregistré les températures de ces ruches sur plusieurs semaines en introduisant la sonde d'un thermomètre dans le couvain et une autre dans la hausse.

a) La ruche A (fig. 19).

La courbe type des enregistrements à l'intérieur de la ruche $A$ nous permet de constater qu'au moment où la température dans la hausse oscille 
entre $20^{\circ}$ (la nuit) et $40^{\circ}$ (le jour) en janvier (petite saison sèche), celle du couvain varie entre $30^{\circ}$ et $38^{\circ}$. Notons que la courbe correspondant aux variations thermiques du couvain reproduit assez fidèlement celle qui dessine les variations externes : c'est dire que la moindre variation climatique extérieure à la ruche, même rapide et de brève durée, se répercute immédiatement dans l'atmosphère qui entoure le couvain.

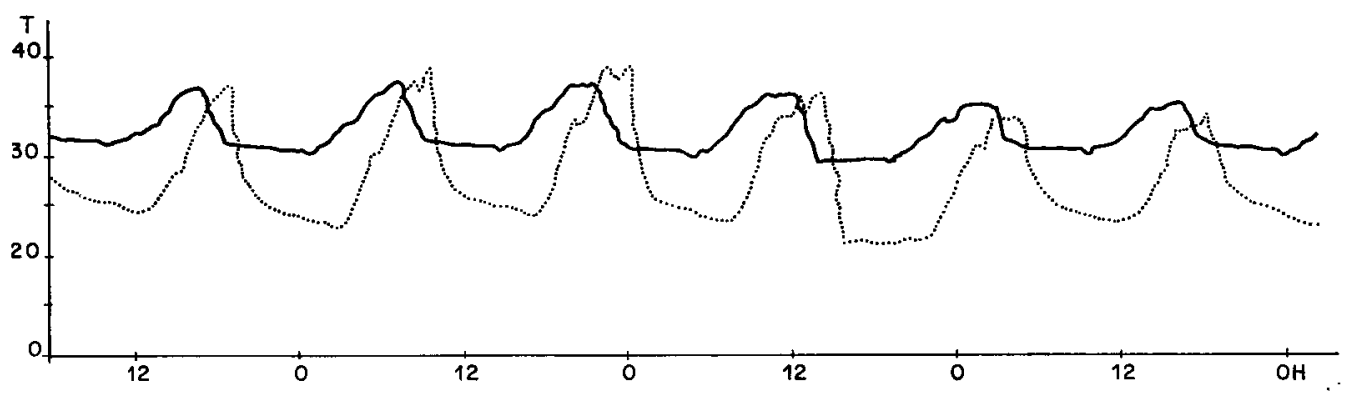

FIG. 19. - Variations thermiques à l'intérieur d'une ruche d'Apis mellifica var. adansonii durant plusieurs jours mesurées au moyen d'une sonde située dans le nid de couvain (trait plein) et dans la hausse (pointillê). La ruche se trouvait dans une clairière parmi des graminées.

Aвв. 19. - Wärmeschwankungen im Innern einer Beute von Apis mell. var. adansonii, während mehrerer Tage mit Hilfe einer Sonde im Brutnest gemessen (ausgezogene Linie) und im Aufsatz (punktierte Linie). Das Volk stand zwischen Gräsern in einer Lichtung.

Il est bon ici de rapprocher ces résultats de ceux obtenus par LENSKY en Isrä̈l dans une serre sous un climat subtropical avec Apis mellifica var. ligustica. On y découvre que, durant une journée, la température externe autour de la ruche peut aller de $22^{\circ}$ à $50^{\circ}$, alors que celle du couvain reste entre $34^{\circ}$ et $38^{\circ}$. Ainsi pour des variations thermiques extérieures et expérimentales plus importantes, les oscillations de température du couvain sont moindres en Israël qu'en Afrique. Il est donc évident qu'à l'instar de ses cousines africaines (Trigones), la variété adansonii possède de moins grandes capacités de régulations thermiques qu'Apis mellifica var. ligustica et certainement que nos autres abeilles européennes, témoin l'une des nombreuses courbes recueillies par BüDEL.

b) La ruche $\mathrm{B}$.

Les deux courbes de la ruche (B) sont doublement intéressantes caı elles nous donnent la possibilité d'apprécier la capacité de thermorégulation d'une même colonie, à la même époque (la petite saison sèche), la même année 1967, au même emplacement mais dans deux situations de biotope très différentes!

En effet, au début des expériences, la colonie se trouvait dans une bananeraie sur le point de donner ses régimes; à la fin, une tornade était passée sur le village, avait déplacé les toits de plusieurs maisons et renversé une partie de la plantation; la ruche était alors à peine protégée par quelques feuilles de bananiers déchiquetées. 
Sur la première courbe (fig. 20), les variations thermiques données par la sonde située dans la hausse sont nettement moindres que celles de la ruche précédente située en plein soleil parmi les graminées : elle n'est en effet que $d^{\prime}$ 'une dizaine de degrés centigrades $\left(18^{\circ}\right.$ à $\left.28^{\circ}\right)$. La courbe fournie par la seconde

T

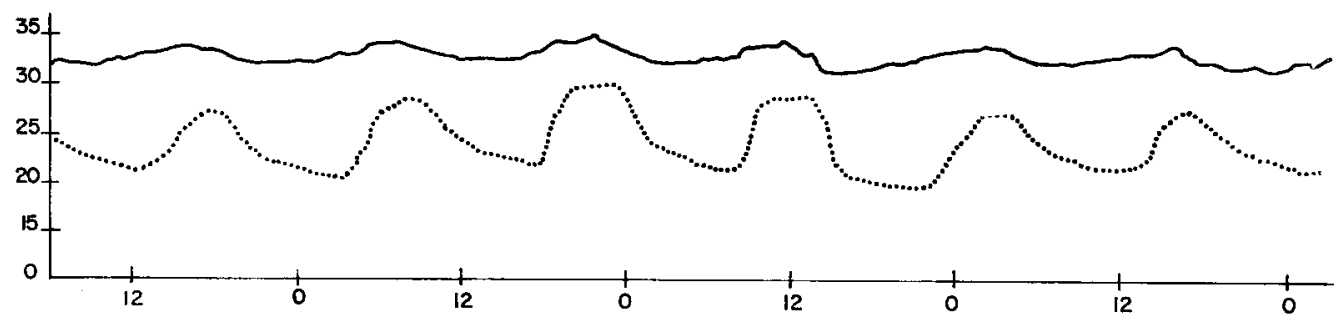

H

Fig. 20. - Courbes d'enregistrement de températures à l'intérieur d'une ruche d'Apis mellifica var. adansonii durant une semaine. Une sonde êtait installée dans le nid de couvain (trait plein) et l'autre dans la hausse (pointillé). La ruche vivait dans une bananeraie prête à donner ses régimes. (Makokou 30-1/6-2-1967).

Aвв. 20. - Die im Innern eines Volkes von Apis mell. var. adansonii während einer Woche gemessenen Temperaturen. Eine Sonde war ins Brutnest eingefüht (ausgezogene Linie), eine zweite befand sich im Aufsatz (punktierte Linie). Das Volk stand in einer Bananenpfanzung kurz vor Erscheinen der Bananentrauben.

sonde placée dans le couvain ressemble presque à celle que l'on retrouve dans les ruches européennes d'Apis mellifica: il n'y a en effet autour de $30^{\circ}$ que $2^{\circ}$ à $3^{0}$ d'écart entre les maxima et les minima. Le biotope particulier favorisait donc la thermorégulation de la ruche.

Après le passage de la tornade (deuxième courbe) (fig. 21), le profil des

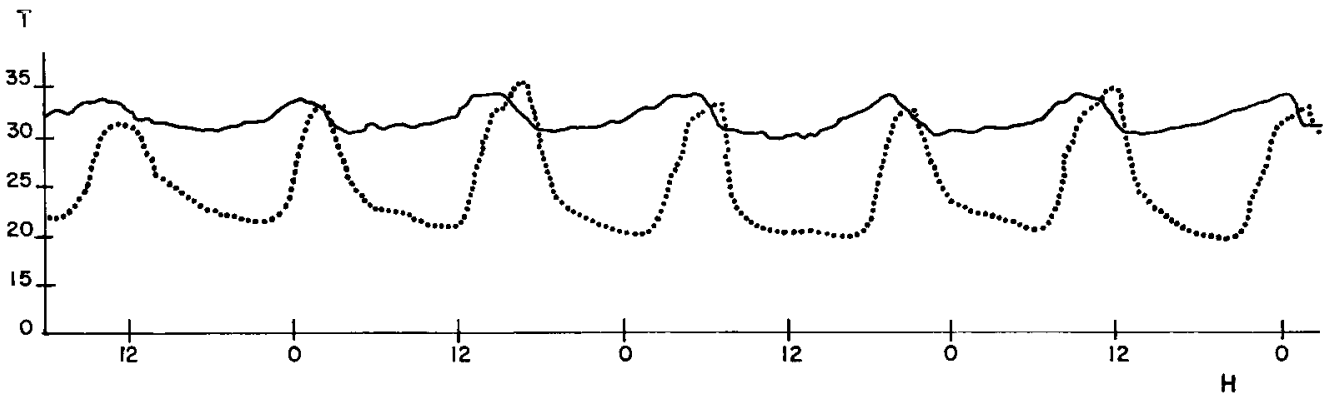

FIG. 21. - Oscillations thermiques dans le couvain (trait plein) et dans la hausse (pointillé) d'une ruche $d$ 'Apis mellifica var. adansonii située dans une bananeraie en partie détruite par une tornade. La colonie d'abeilles est la même que celle qui nous a permis d'obtenir la courbe précédente (Fig. 20).

Авв. 21. - Wärmeschwankungen im Brutnest (ausgezogene Linie) und im Aufsatz (punktiert) eines Volkes von Apis mell. var. adansonii in einer Bananenpflanzung, die teilweise durch einen Tornado zerstört war. Das Volk ist das gleiche wie in Abb. 20.

courbes est beaucoup plus tourmenté : les températures « extérieures » sans atteindre les maxima d'une ruche exposée au soleil atteignent tout de même $36^{\circ}$, et les oscillations de températures du couvain sont de l'ordre de $5^{\mathbf{0}}$.

En résumé, 1) Apis mellifica adansonii assure une climatisation de son habitat beaucoup plus imparfaite que celle de nos abeilles d'Europe, 2) cette 
variété semble cependant réagir beaucoup mieux aux basses températures qu'aux températures élevées. Très inférieure à la variété ligustica dans les pays chauds, elle se montre presque équivalente à nos mellifica sous des climats tempérés.

\section{2. - La nidification d'Apis mellifica adansonii en Afrique}

Les capacités régulatrices de cette variété permettent de comprendre sa nidification dans les zones forestières ou savanicoles que nous avons étudiées. On la rencontre en effet dans ces deux milieux. En savane, à défaut de troncs d'arbres creux, elle sait devenir terricole et coloniser les cavités creusées par les animaux dans le système radiculaire des palmiers rôniers adultes (Borassus aethiopum) dont les palmes s'étendent facilement à une vingtaine de mètres au-dessus du sol. En forêt gabonaise dans les clairières découpées par de jeunes plantations de manioc elles introduisent leurs rayons de cire parmi les racines d'arbre parcourant de petits tertres ou bien des termitières abandonnées (fig. 22). Mais les colonies sont en général très petites (la variété adansonii essaime beaucoup) et les rayons de cire sont peu nombreux.

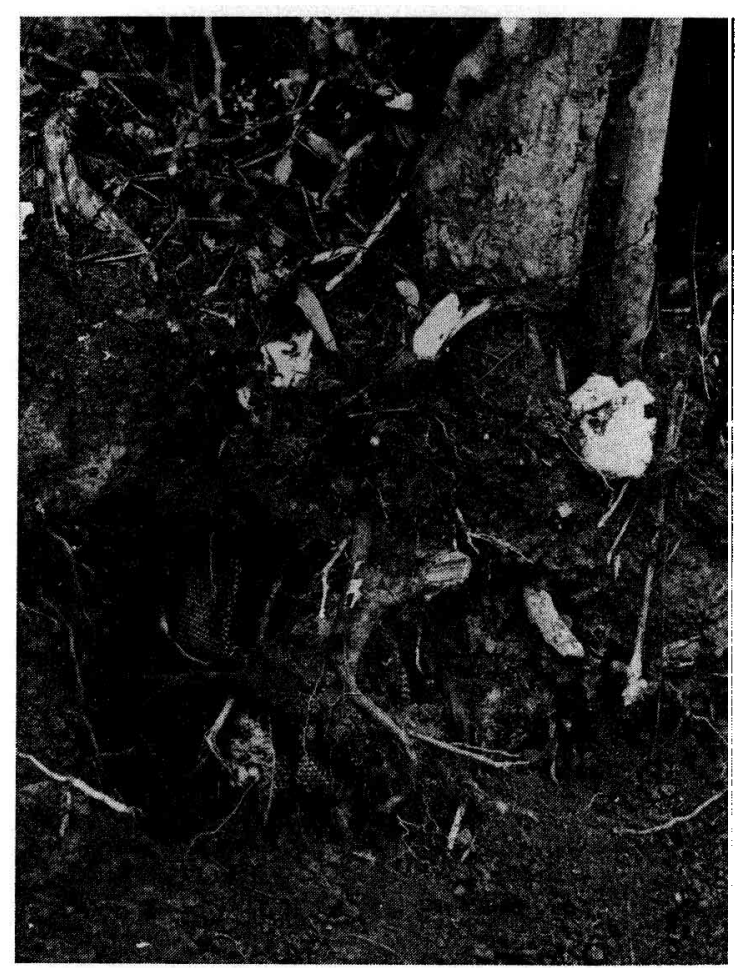

Fig. 22. - Coupe d'un nid terricole d'Apis mellifica var. adansonii à Makokou (Gabon). Aв8. 22. - Schnitt durch ein Erdnest von Apis mell. var. adansonii in Makakou (Gabun). 


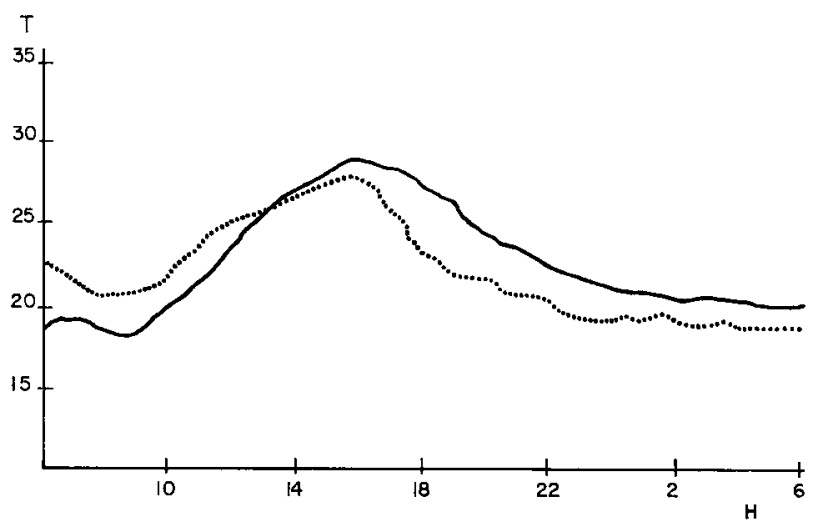

Fig. 23. - (d'après ZucchI et SAKagami). Variations thermiques d'un nid de Trigona spinipes au Brésil. Le trait plein représente la courbe de température à l'intérieur du couvain, les pointillés celle de l'air ambiant.

Aв8. 23. - (nach ZucchI u. Sakagami). Wärmeschwankungen in einem Nest von Trigona spinipes in Brasilien. Die ausgezogene Linie gibt die Temperatur im Innern des Brutnestes an, die punktierte Linie die der umgebenden Luft.

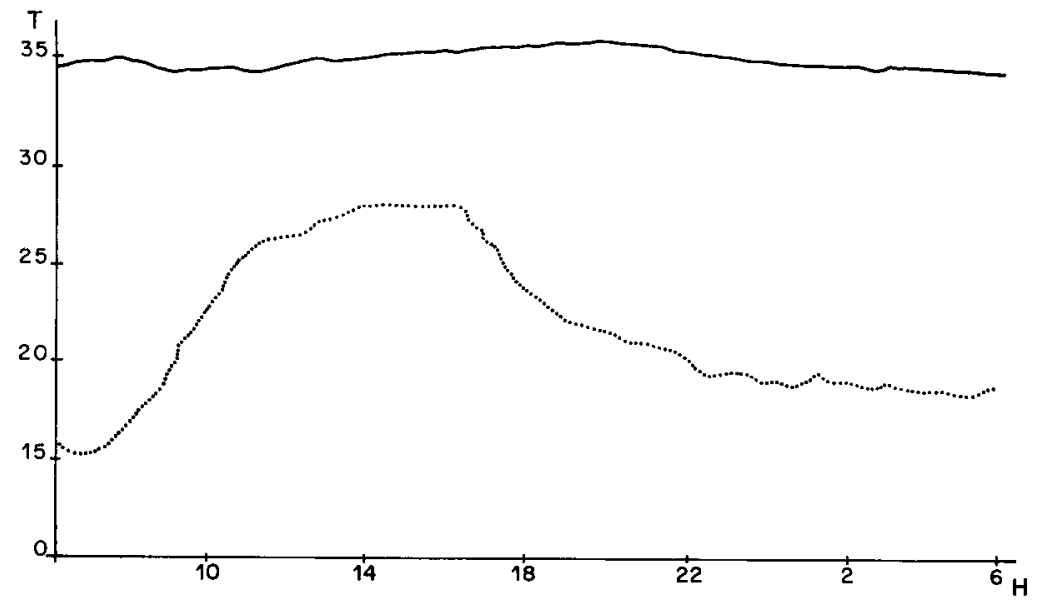

Frc. 24. - (d'après ZuCChI et SAKagami). Profil des variations thermiques d'un nid de Leurotrigona mulleri au Brésil. Les thermomètres étaient fichés dans le couvain (trait plein), dans l'écorce du nid (pointillé).

Aвв. 24. - (nach ZuCCHI u. SAKaGaMI). Wärmeschwankungen in einem Nest von Leurotrigona muelleri in Brasilien. Die Thermometer waren im Innern des Brutnestes (ausgezogene Linie) und an seinem Rande angebracht (punktierte Linie). 


\section{VI. - CONCLUSIONS}

Zucchi et Sakagami ont récemment étudié le même problème chez Trigona spinipes et d'autres espèces de Trigones d'Amérique du Sud. Eux aussi comme moi en 1967 avec Trigona nebulata et en 1966 avec Dactylurina staudingeri ont constaté les diverses aptitudes de thermorégulation des différentes espèces de Trigones : elle va d'une remarquable capacité de climatisation chez Trigona spinipes à une incapacité presque totale chez Leurotrigona mülleri. Chez la première espèce, la température du couvain varie par exemple entre $33^{0}, 3$ et $36^{0}, 2$ pendant que la température ambiante oscille entre $8^{0}, 2$ et $30^{\circ}, 3$. Chez la dernière au contraire, la température du couvain passe aisément de $16^{0}, 5$ à $29^{\circ}, 5$ tandis que le thermomètre installé à l'extérieur de la ruche va de $15^{0}, 5$ à $28^{\circ}$. Les figures ( 23 et 24 ) extraites du travail des auteurs précédents présentent clairement les aptitudes variables de thermorégulation des différentes espèces étudiées. Or il est remarquable que la structure du nid de Trigona spinipes ressemble assez à celle de Dactylurina staudingeri et que Leurotrigona mülleri comme Trigona nebulata profite de l'hospitalité des Termites. Il semble donc que les abeilles qui construisent en totalité leurs nids se caractérisent par une réelle aptitude thermorégulatrice, alors que les abeilles termitophiles ou myrmécophiles s'en trouvent presque privées.

Les auteurs précédents ne nous fournissent malheureusement pas de renseignements sur le biotope et l'écologie de chacune des espèces américaines qu'ils ont étudiées. La comparaison de nos observations auraient alors été certainement très instructive.

Il serait intéressant, en outre, de reprendre ces travaux sur la thermorégulation d'Apis mellifica adansonii en Amérique du Sud où cette espèce a été malencontreusement importée. A-t-elle réussi à acquérir des aptitudes thermorégulatrices égales à celles des abeilles européennes? Quels sont les biotopes préférés de ces insectes dans cette région du globe? 


\section{ZUSAMMENFASSUNG}

1. Wir berichten über Beobachtungen und Versuche, die in einigen Gegenden Afrikas durchgeführt wurden und die die Wärmeregulierung in den Bienenstöcken verschiedener afrikanischer sozialer Bienen betreffen. An den untersuchten Arten stellen wir fest, dass die Tiere mit einer mehr oder weniger ausgeprägten Fähigkeit auf die physikalischen Bedingungen des Nestklimas einwirken. Bestimmte Bienen können das Mikroklima ihrer Behausung regulieren und optimale Temperaturen für die Entwicklung erreichen, andere dagegen sind unfähig, eine wirksame Regelung sicherzustellen. Die Regulierung des Mikroklimas ist oft das Ergebnis mehrerer zusammenhängender Handlungen. Wir haben uns besonders mit den Änderungen beschäftigt, die die Bienen im Tages- und Nachtablauf an der Architektur ihres Nestes ausführen. Bei dieser ersten Aufgabe wurden die verschiedenen Modalitäten des Nestbau-Verhaltens untersucht. So haben wir über die Wahl des Ortes für den Nestbau bei den herrschenden klimatischen Bedingungen berichtet und konnten eine enge Beziehung zwischen diesen beiden Faktoren feststellen. Dadurch wird auch verständlich, wieso man Bienen in derart verschiedenen Gegenden wie die Savanne der Elfenbeinküste und die Wälder von Gabun findet. Das Problem der Wärmeregulierung des Bienenstockes ist für Apis mellifica, var. adansonii auf's beste analysiert worden. Wir besitzen über diese Biene zahlreiche vergleichende Arbeiten aus Ländern der gemässigten Zone und zwar von verschiedenen Varietäten der gleichen Art.

2. Um die Gebiete, in denen wir gearbeitet haben, in klimatologischer Hinsicht besser zu umreissen, geben wir einige Querschnitte der thermischen und hygrometrischen Aufzeichnungen in den hauptsächlichen Biotopen. In der Savanne der Elfenbeinküste weisen die Kurven sehr deutliche Schwankungen auf, die bedeutende Unterschiede zwischen den Maxima und Minima aufzeigen. In Gabun, in den hier und da im Innern des Waldes genutzten Kahlschlägen verhält es sich ebenso. Dagegen weisen die Galeriewälder, die die Savanne umsehliessen, weniger schwankende Temperaturen und gleichbleibende Feuchtigkeit auf; und im dichten äquatorialen Wald sind Temperatur und Feuchtigkeit bemerkenswert beständig.

3. Um einwandfrei leben zu können, brauchen die Bienen eine Wohnung, deren Temperatur wenig schwankt. Den Arten entsprechend wird dieses unerlässliche thermische Gleichgewicht auf verschiedene Art und Weise aufrechterhalten. Die Arten, denen wirksame regulatorische Fähigkeiten fehlen, überleben und gedeihen dank einer sorgfältigen Auswahl ihrer Nistplätze. Sie bleiben dem Wald verhaftet, der ihnen ein ausgeglichenes Klima gewährleistet, und selbst dort präzisieren sie ihre Anforderungen und nisten an solchen Stellen, an denen das Ausmass der Wärmeschwankungen, denen sie ausgesetzt sein könnten, verringert ist. Das trifft für Trigona beccarii und Trigona nebulata zu. Die Unfähigkeit der letzteren, die Nestwärme zu regeln, wurde mit Hilfe einer Sonde bewiesen, die mitten ins Brutnest eingeführt wurde. Meliponula bocandei ist ebenfalls eine im Walde lebende Art. Ihr Verbreitungsgebiet ist ziemlich gross, doch das führt zu noch grösserer Sorgfalt bei der Wahl des Nistplatzes. Diese Art lebt da und dort, aber in ökologisch vergleichbarer Umgebung. Diese Tatsache ist nicht immer auf Anhieb zu erkennen, so dass man geneigt sein könnte, ihre Fähigkeiten zu überschätzen.

4. Haben die von uns angeführten Bienen nur geringe oder gar keine Möglichkeiten der Wärmeregulierung, so gibt es andere, die besser mit ihrer Umgebung fertig werden, weil sie eine Summe von Anpassungsfähigkeiten entwickelt haben, um das Mikroklima ihres Nestes zu regeln. Diese Fähigkeiten weisen zwei Gruppen auf : die Hypotrigonen und die Dactylurinen. Trotzdem kommt auch hier der Wahl des Nistplatzes eine gewisse Bedeutung zu; doch sie ist nicht mehr allein bestimmend in bezug auf die Möglichkeit der Artenverbreitung. Tatsächlich ändert sich der Bau des Nestes den Biotopen entsprechend, wofür Dactylurina 
in der Sevanna und im Wald besonders Zeugnis ablegt. Aber darüber hinaus ist das Verhalten der Tiere auf die Notwendigkeit der Klimatisation eingestellt. Man kann Bienen sehen oder gar hören, die stundenlang Luft in den Stock fächeln und man kann beobachten, wie die Nestumhüllung durchlöchert wird, um die Luftzirkulation zu erleichtern. Bei den Arten, die in der Savanne leben, gibt es höchstwahrscheinlich zu gewissen Zeiten auch eine Wasserversorgung.

5. Im Vergleich zu diesen Arten, bei denen die Wärmeregelung gesichert ist, wurde unter den gleichen Gesichtspunkten Apis mellifica var. adansonii untersucht. Wir wissen, dass die europäische Biene fähig ist, eine ausgezeichnete, gleichbleibende Wärme im Stock sicherzustellen. Nun, in dieser Beziehung versagt die afrikanische Varietät einigermassen. Dies erklärt vielleicht auch gewisse ungewöhnliche Plätze von Nestanlagen. Tatsächlich finden wir sie manchmal in den Nestern baumbewohnender Ameisen, in Termitenhügeln oder selbst in unterirdischen Kammern. Diese Biene scheint ihre grosse Verbreitung in Afrika ihrer Vielseitigkeit in der Wahl des Nistplatzes zu verdanken, einer Vielseitigkeit, die nur durch die herrschenden Wärmebedingungen beschränkt wird.

In unserer Arbeit weisen wir schliesslich noch auf die Parallelität zwischen unseren Ergebnissen und den von ZUCCHI und SAKAGAMI bei südamerikanischen Trigonen-Arten gewonnenen hin. Auch diese Autoren haben verschiedene Fähigkeiten der Wärmeregulierung festgestellt, je nach der untersuchten Art. Einige von ihnen können praktisch ebenso wie gewisse afrikanische Arten die Isothermie im Stock aufrechterhalten, während andere Fähigkeiten besitzen, die in der gleichen Weise entwickelt sind wie bei Apis mell. in Europa. Beim jetzigen Stand unserer Kenntnisse wäre es sehr interessant, die Ökologie von Apis mell. var. adansonii in Amerika zu untersuchen und herauszufinden, ob diese Art auf dem neu erworbenen Kontinent bessere Fähigkeiten der Wärmeregelung entwickeln konnte.

\section{REFERENCES BIBLIOGRAPHIQUES}

Brosset A., Darchen R., 1967. Une curieuse succession d'hôtes parazites des nids de Nasutitermes. Biologia gabonica, 4, 153-168.

Büdel A., 1968. Le microclimat de la ruche. In : “ Traité de Biologie de l'Abeille », Masson, Paris.

Darchen R., 1965. Ethologie d'une Araignée sociale. Agelena consociata Denis. Biologia gabonica, 1, 2, 117-146.

Darchen R., Pain J., 1966. Le nid de Trigona (Dactylurina) staudingeri grib. (Hyménoptère : Apidae.) Biologia gabonica, 2, 1, 25-35.

Darchen R., 1966. Sur l'Ethologie de Trigona (Dactylurina) staudingeri grib. (Hyménoptère : Apidae). Biologia gabonica, 2, 1, 37-45.

Darchen R., 1969. Sur la biologie de Trigona (Apotrigona) nebulata Komiensis Cok. I. Biologia gabonica, 5, 3, 151-187.

Darchen R., 1971. Trigona (Axestotrigona) oyani Darchen (Apidae, Trigoninae), une nouvelle espèce d'abeille africaine. Description du nid inclus dans une fourmilière. Biologia gabonica, 4, 407-421.

Josens G., 1971. Variations thermiques dans les nids de Trinervitermes geminatus wasmann en relation avec le milieu extérieur dans la savane de Lamto (Côte-d'Ivoire). Insectes Sociaux, 18, 1-13.

Lenskx Y., 1963. Études sur la physiologie et l'écologie de l'Abeille (Apis mellifica L. var. ligustica) en Israël. Thèse. 93 p. Université Hébraĩque de Jérusalem.

Lindauer M., 1954. Temperaturregelung und Wasserhaushalt in Bienenstaat. Z. Vergl. Physiol., 36, 391-432. 
MÉNager M.-T., 1970. Étude climatologique au contact de la forêt et de la savane (Lamto). Bull. Liaison Chercheurs Lamto, juillet, 5-10.

Nogueira-Neto P., 1948. Notas bionômicas sôbre meliponineos. I. Sôbre a ventilaçâo dos ninhos e as construçôes comela relacionadas. Rev. Brasil. Biol., 8, 465-488.

Tournier J.-L., 1970. Fiches climatologiques, Bull. Liaison Chercheurs Lamto, novembre, 2-10.

Tournier J.-L., Monnier Y., Ménager M.-T., 1970. Le climat. Bull. Liaison Chercheurs Lamto, Numéro Spécial, $2^{\mathrm{e}}$ partie, 40-59.

Zucchi R., Saxagami S. F., 1972. Capacidade termo-reguladora em Trigona spinipes e em algumas outras espécies de abelhas sem ferrâo (Hymenoptera : Apidae : Meliponinae). In : “ Homenagem d̀ W. E. KERR », Rio Claro, Brésil. 